\title{
Haptic Object Localization in the Vibrissal System: Behavior and Performance
}

\author{
Per Magne Knutsen, Maciej Pietr, and Ehud Ahissar \\ Department of Neurobiology, The Weizmann Institute of Science, 76100 Rehovot, Israel
}

Using their large mystacial vibrissas, rats perform a variety of tasks, including localization and identification of objects. We report on the discriminatory thresholds and behavior of rats trained in a horizontal object localization task. Using an adaptive training procedure, rats learned to discriminate offsets in horizontal (anteroposterior) location with all, one row, or one arc of whiskers intact, but not when only a single whisker (C2) was intact on each cheek. However, rats initially trained with multiple whiskers typically improved when retested later with a single whisker intact. Individual rats reached localization thresholds as low as $0.24 \mathrm{~mm}\left(\sim 1^{\circ}\right)$. Among the tested groups, localization acuity was finest $(<1.5 \mathrm{~mm})$ with rats that were initially trained with all whiskers and then trimmed to one arc of whiskers intact. Horizontal acuity was finer than the typical inter-vibrissal spacing $(\sim 4.8 \mathrm{~mm}$ at contact points). Performance correlated with the net whisking spectral power in the range of $5-25 \mathrm{~Hz}$ but not in nonwhisking range of $30-50 \mathrm{~Hz}$. Lesioning the facial motor nerves reduced performance to chance level. We conclude that horizontal object localization in the rat vibrissal system can reach hyperacuity level and is an active sensing process: whisker movements are both required and beneficiary, in a graded manner, for making accurate positional judgments.

Key words: rats; whisking; active touch; hyperacuity; learning; sensory deprivation

\section{Introduction}

The array of mystacial vibrissas (whiskers) is an important tactile sensory apparatus in many mammals. Rats can use their whiskers to discriminate textures (Vincent, 1912; Guic-Robles et al., 1989; Carvell and Simons, 1990), localize objects (Brecht et al., 1997; Harvey et al., 2001), judge distances (Jenkinson and Glickstein, 2000), discriminate widths of apertures (Krupa et al., 2001; Shuler et al., 2002), and discriminate surface orientations (Polley et al., 2005). The mystacial pad of some rodents contains musculature specialized for controlling whisker movement (Dorfl, 1982; Wineski, 1985; Berg and Kleinfeld, 2003a), which allows the whiskers to protract and retract along the posteroanterior axis (Wineski, 1983; Bermejo et al., 1998; Sachdev et al., 2002). Variations in whisking amplitudes $\left(<70^{\circ}\right)$ and frequencies $(5-25 \mathrm{~Hz})$ have led observers to suggest that whisking kinematics is tuned to task requirements (Wineski, 1983; Carvell and Simons, 1990; Harvey et al., 2001; Berg and Kleinfeld, 2003a; Sachdev et al., 2003). However, Krupa et al. (2001) found that whisking is not required to localize objects along the radial dimension (from the

\footnotetext{
Received April 8, 2006; revised June 7, 2006; accepted July 6, 2006.

This work was supported by Israel Science Foundation Grant 377/02-1, The Minerva Foundation funded by the Federal German Ministry for Education and Research, Human Frontiers Science Program RG0043/2004, and the German Federal Ministry of Education and Research-Israeli Ministry of Science and Technology Foundation. E.A. holds the Helen Diller Family Professorial Chair of Neurobiology. We thank Alon Fichman, Marcin Szwed, Naama Rubin, Sebastian Haidarliu, and Knarik Bagdasarian for their assistance throughout these experiments; David Kleinfeld, Samar Mehta, Mathew Diamond, and Shabtai Barash for their critical comments; and Barbara Schick for reviewing this manuscript.

Correspondence should be addressed to Per Magne Knutsen, Department of Neurobiology, The Weizmann Institute of Science, 76100 Rehovot, Israel. E-mail: per.knutsen@weizmann.ac.il.

DOI:10.1523/JNEUROSCI.1516-06.2006

Copyright $\odot 2006$ Society for Neuroscience $\quad$ 0270-6474/06/268451-14\$15.00/0
}

cheek and outwards); performance in such a task depends on the number (but not the identity) of intact whiskers. Thus, the additional temporal information about contacted objects and surfaces conveyed by the kinematics of a moving sensory array does not appear beneficiary in all tasks, and the exact behavioral relevance of whisking kinematics remains elusive.

Across modalities, sensor movements can improve performance in spatial tasks. Fixational eye movements are important for visual processes (Kelly, 1979; Tulunay-Keesey and VerHoeve, 1987; Rucci and Desbordes, 2003), head movements may assist in sound localization (Wallach, 1940; Perret and Noble, 1997; Wightman and Kistler, 1999), and hand movements improve tactile performance (Lamb, 1983; Gamzu and Ahissar, 2001). Movements of eyes and hands move stimuli across sensory receptors, thus enriching sensation with information unavailable when stimuli are fixed in place on the eye or hand (Ahissar and Arieli, 2001). Similarly, whisker movements enrich the tactile world perceived by the rat. For instance, during some texture discrimination tasks, rats appear to sweep their whiskers across textured surfaces at velocities that optimize encoding by cutaneous receptors (Carvell and Simons, 1990).

Here, we investigated the role of whisker movements during localization of objects along the horizontal (posteroanterior) axis. We showed that rats actively move their whiskers during localization and found that whisking is indeed required and beneficiary, in a graded manner, for accurate horizontal localization. Discrimination in our task occurred in a brief temporal window (400-600 ms, approximately four contacting whisks), and performance thresholds extended down to approximately $1 / 10$ of the typical inter-vibrissal spacing. Our behavioral results show that horizontal object localization in the rat vibrissal system is an 
Table 1. Experimental groups and learning trends

\begin{tabular}{|c|c|c|c|c|c|c|}
\hline Intact whiskers & Rats $^{a}$ & Sessions $^{b}$ & $S-80^{c}$ & $R_{\text {pre }} / R_{\text {post }}{ }^{d}$ & $P_{\text {pre }}^{e}$ & $S D_{\text {pre }} / S D_{\text {post }}^{f}$ \\
\hline ALL & $8(4)$ & $11 \pm 7$ & $3.7 \pm 1.4$ & $-0.89 /-0.39$ & $<0.01$ & $33 / 20$ \\
\hline $\mathrm{ALL} \triangleright \mathrm{C} 2$ & 4 & $15 \pm 8$ & $2.8 \pm 1.0$ & $-0.89 / 0.36$ & $<0.01$ & $34 / 21$ \\
\hline$A L L \triangleright A R C$ & 4 & $8 \pm 1$ & $3.0 \pm 0.8$ & $-0.98 / 0.16$ & $<0.001$ & $26 / 12$ \\
\hline $\mathrm{ALL} \triangleright \mathrm{ARC} \triangleright \mathrm{C}$ & 3 & $11 \pm 2$ & $1.7 \pm 0.6$ & -10 & & $36 / 24$ \\
\hline ROW & $6(0)$ & $11 \pm 5$ & $2.5 \pm 1.0$ & $-0.94 /-0.35$ & $<0.01$ & $26 / 21$ \\
\hline $\mathrm{ROW} ・ \mathrm{C} 2$ & 3 & $11 \pm 4$ & $2.3 \pm 1.5$ & $-0.23 / 0.05$ & NS & $21 / 20$ \\
\hline ROW $\triangleright \mathrm{C} 2 \triangleright$ NONE & 2 & $9 \pm 0$ & $6.5 \pm 0.7$ & $0.39 / 0.96$ & NS & $24 / 26$ \\
\hline ARC & $6(1)$ & $14 \pm 6$ & $4.2 \pm 1.0$ & $-0.83 /-0.28$ & $<0.01$ & $27 / 22$ \\
\hline $\mathrm{ARC} \triangleright \mathrm{C} 2$ & 3 & $12 \pm 6$ & $3.3 \pm 3.2$ & $-0.45 /-0.22$ & NS & $19 / 23$ \\
\hline $\mathrm{ARC} / \mathrm{ROW} \bullet \mathrm{FN}^{\mathrm{g}}$ & 3 & $8 \pm 1$ & $1 \pm 0$ & $-/-0.15$ & & $-/ 27$ \\
\hline $\mathrm{C} 2$ & $6(3)$ & $13 \pm 8$ & $5.8 \pm 4.7$ & $-0.78 /-0.15$ & $<0.05$ & $27 / 25$ \\
\hline ALL (NAIVE) & 3 & $6 \pm 3$ & & & & \\
\hline
\end{tabular}

${ }^{a}$ Rats successfully (unsuccessfully) trained in each condition.

${ }^{b}$ Number of training sessions (mean \pm SD; not including preceding conditions).

'Session number in which performance threshold reached $\geq 80 \%$ of best performance. For each rat, thresholds of successive training sessions were normalized to the best performance (mean \pm SD).

${ }^{d}$ Regressions of threshold and session number before (pre) and after (post) the S-80 session (mean \pm SD). Not estimated when S-80 was $<2$.

${ }^{e}$ Probabilities that $R_{\text {pre }}$ was generated without improvement in performance. None of the $R_{\text {post }}$ regressions were significant.

'Variability of performance percentage of a rat's best performance for sessions before (pre) and after (post) the S-80 session (mean \pm SD).

${ }^{g}$ Facial nerve-lesioned rats.

active process, which is tuned to the available sensory array, and that it can be improved to a hyperacuity level by selective sensory deprivation.

\section{Materials and Methods}

Animals. Thirty-four male albino rats (Wistar) were trained in a two-alternative forced-choice task to discriminate the relative horizontal offset of two vertical poles. Training commenced at the age of 7 weeks. Rats were housed two in a cage on a $12 \mathrm{~h}$ light/dark cycle. Individual training sessions (45 min) commenced in the early evening, coinciding with an endogenous rise in exploratory activity, and were repeated two or three times a week. Water was withheld for $20-24 \mathrm{~h}$ before each training session. Fruit juice (mango-flavored) was given as reward during training, and unlimited water was available throughout the following 20-24 h. Solid food was available ad libitum, except during training.

Rats were initially trained with different sets of whiskers intact. One group initially had all whiskers intact, and other groups had an arc, a row, or a single pair of whiskers intact ( $\operatorname{arc} 2$, row $\mathrm{C}$, and $\mathrm{C} 2$, respectively) on both cheeks. For some groups, the configuration of intact whiskers was changed as the behavioral testing progressed. For example, rats with initially all whiskers intact (the ALL group), later had some of their whiskers clipped and were retested with a single whisker intact on each cheek (ALL-C2). Testing conditions are shown in Table 1. When whiskers were clipped, they were clipped as close as possible to the skin $(<1 \mathrm{~mm})$ during brief (5-10 $\mathrm{min})$ isoflurane anesthesia; this procedure was repeated three times each week. Animal maintenance and all experimental procedures were conducted in accordance with National Institutes of Health and The Weizmann Institute of Science guidelines.

Behavioral apparatus. Rats were trained and tested in a $25 \times 40 \mathrm{~cm}$ behavioral box (Fig. 1). The box had three openings, one on each long side and one in the front. The openings on the sides provided access to drinking wells where the reward was presented. Attempts to retrieve rewards from the wells were detected by an infrared beam (E24-01 Optical Lickometer; Coulbourn Instruments, Allentown, PA). Solenoid pinch valves (360P012-42; NResearch, West Caldwell, NJ) ensured accurate control of aliquot sizes. The opening at the front of the box provided access to an area where discriminanda were presented during trials.

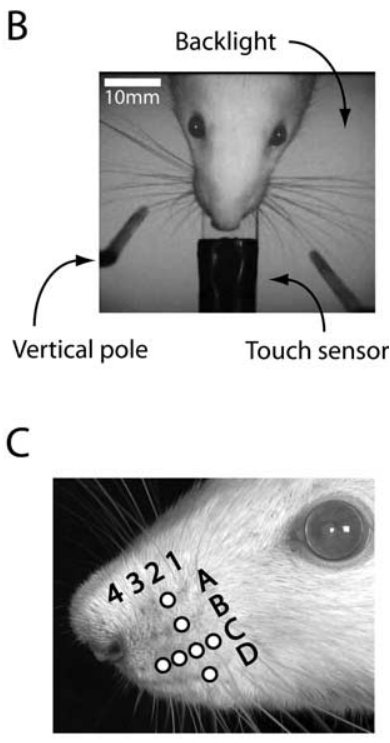

Backlit discrimination area

Figure 1. Behavioral apparatus. $\boldsymbol{A}$, Configuration of the behavioral box and its components. The horizontal locations of the poles (distance from the wall of the box) were changed between trials. $\boldsymbol{B}, 0$ bjects in the discrimination area as they appear in video frames. $C$, The mystacial pad area of the rat, with row $C$ and arc 2 indicated.

Movements through each opening were detected by an infrared beam. Access to all openings was controlled by movable doors (HS-300; Hitec, Poway, CA). Only the head of the rat could go through the front opening. In the discrimination area, there were four objects that were within reach of the rat. A flat piece of Perspex $(1 \mathrm{~cm}$ wide $\times 10 \mathrm{~cm}$ long $)$ was attached to the outside wall of the behavioral box and prevented downward head movements. A circular ( $1 \mathrm{~cm}$ diameter) nose poke, connected to a touchsensitive capacitance switch (F53N; David Johnson \& Associates, Thornton, $\mathrm{CO}$ ) was located $8 \mathrm{~cm}$ from the box. A movable, vertical metal pole ( $2.8 \mathrm{~mm}$ diameter) was located on either side of the nose poke and was attached to a linear motor with micrometer resolution (Abiry, Tel Aviv, Israel). Two infrared $(880 \mathrm{~nm}$ ) light-emitting diode (LED) spotlights (F54845; Edmund Industrial Optics, Barrington, NJ), which were located $10-15 \mathrm{~cm}$ above the nose poke, produced visible reflections in the eyes of the rat, thus facilitating tracking of head movements. A high-speed camera [ 500 frames per second (fps)] was placed $\sim 50 \mathrm{~cm}$ above the discrimination area (MotionScope PCI; Redlake, San Diego, CA), and an additional infrared sensitive CCD camera (55-701; Edmund Industrial Optics) was also positioned above the behavioral box. A backlight, made 
from a $10 \times 10$ array of infrared (940 nm) LEDs (L940-04AU; Epitex, Kyoto, Japan), was placed $\sim 10 \mathrm{~cm}$ below the discrimination area, which improved visualization of the whiskers. Behavioral training sessions were entirely computer controlled, and all events (crossing of infrared beams, control of valves and motors, and video capture) were logged with millisecond resolution.

Behavioral training. Training, which was preceded by $1 \mathrm{~d}$ of acclimatization, consisted of two phases: procedural and discriminative. During procedural training, rats acquired associations between the reward, its location, and actions that triggered its availability. At the end of procedural training, and throughout discriminative training, a rat voluntarily placed its head within the discrimination area and contacted the nosepoke touch sensor in return for reward. After touching the nose poke, the rat voluntarily withdrew from the discrimination area and approached one of the two reward wells. If the rat made a correct association between the reward location and the positions of the poles, it was rewarded with fruit juice. If incorrect, no reward was given. After a variable delay (20-30 s), this trial procedure was repeated. Our training protocol was adapted and modified from that of Krupa et al. (2001).

Procedural training. Initial training sessions involved learning of the task procedure without the vertical poles being presented. Procedural training consisted of four stages. In the first stage, the two wells on either side of the box were associated with reward. The front door was kept closed and both side doors were open. When the rat approached any well, a $70 \mu \mathrm{l}$ aliquot of reward was presented. In the second stage, which commenced once the rat consistently approached both wells, a reward was presented on either the left or right side. After the reward was retrieved, a tone $(1.5 \mathrm{kHz})$ sounded and the doors in front of both wells closed. After a variable timeout (5-20 s), one door reopened, thus signaling a new trial. A reward was given if the rat responded within $5 \mathrm{~s}$. In the third stage, the front door was opened at the beginning of the trial, allowing the rat to place its head outside the behavioral box and to explore the discrimination area. The door to one (randomly chosen) reward well was opened when the rat placed its head within the discrimination area. The door emitted a characteristic sound that was used as a signal for reward retrieval. After the reward was retrieved, the front door closed, preventing any additional access to the discrimination area in that trial. After the reward was consumed ( $4 \mathrm{~s}$ delay), the door to the reward well was closed, and a new trial commenced after a variable delay $(10-25$ s). In the last stage, the rat was trained to contact the touch sensor with its nose. Initially, the door of one well opened when the nose of the rat was in close proximity to the touch sensor. The criteria for contact was then gradually refined until the rat actually contacted the touch sensor with its nose. Once a rat successfully learned these stages, discriminative training ensued.

Discriminative training. The discriminanda were two vertical poles, one placed on each side of, and $20 \mathrm{~mm}$ from, the midline (Fig. 1). The poles were repositioned before each trial while the front door of the behavioral box was closed. During repositioning, both poles were initially moved to a location halfway between their final positions and then moved simultaneously and with the same velocity to ensure their arrival at the target locations at the same time. Therefore, acoustics (e.g., pitch or duration) emitted by the motors did not contain information about the relative offset of the poles. The poles were only moved horizontally, parallel to the axis that connected the frontal opening of the behavioral box with the nose-poke sensor. The task of the rat was to judge which of the two poles was more posterior (closer to the front door of the behavioral box).

Discriminative training consisted of two stages. In the first, discriminanda were introduced, and their locations were associated with a reward well. In each of these trials, both side doors opened when a rat contacted the nose poke. The rat then withdrew from the discrimination area, and the door in front of the incorrect well closed while the door in front of the correct well (on the side of the more posterior pole) remained open for the rat to retrieve the reward. During these association trials, rats were always rewarded if the response was made within $5 \mathrm{~s}$ of contacting the nose poke. Once rats responded without delay in association trials, we introduced discrimination trials in which reward was contingent on making a correct response. During initial training sessions, some rats became passive if they made several consecutive errors. Therefore, association and discrimination trials were initially interleaved.

During discrimination trials, both side doors opened when the rat touched the nose poke. A reward was given only when a rat approached the well on the same side as the more posterior pole within $5 \mathrm{~s}$. Thus, if the right pole was more posterior, a reward was given only in the well on the right side of the behavioral box. If a rat did not respond within $5 \mathrm{~s}$ or if it approached the incorrect well, a $1.5 \mathrm{kHz}$ tone signaled the end of the trial, and all of the doors (sides and front) closed. In both association and discrimination trials, rats were allowed $4 \mathrm{~s}$ to consume the reward, followed by a $1.5 \mathrm{kHz}$ tone and closure of all doors. After a random delay (20-30 s), a new trial commenced. Trials were cancelled if either the rat kept its head outside of the behavioral box for longer than $10 \mathrm{~s}$ or it withdrew its head back into the box before touching the nose poke.

Stimulus presentation. The location of the more posterior pole was kept at a constant distance from the wall of the behavioral box, thus acting as a reference signal, or "pedestal" (Green and Swets, 1966), for the position of the anterior pole. In the first trial, the anterior pole was located $20 \mathrm{~mm}$ (more anterior) behind the posterior pole. This distance was reduced or increased after each trial according to the following one-up/one-down staircase rule:

$$
10^{\log _{10} F+\delta},
$$

where the change in offset $(\delta)$ was -0.1 after correct trials and 0.2 after incorrect trials, and $F$ was the offset in the previous trial. The maximal offset was $20 \mathrm{~mm}$, and failures at this offset were followed by another trial with a $20 \mathrm{~mm}$ offset. There was no lower boundary on the offset. With this staircase rule, the success rate would be $\sim 67 \%$ at threshold levels (assuming performance was stable after threshold was reached), because the up step was twice the down step.

The relative offset between the two poles, i.e., which was more posterior, was randomized only in the first 10 trials and chosen according to the following bias-correcting rules in subsequent trials. These rules took into account the responses that each rat made during the 10 preceding trials:

$$
\begin{aligned}
& |S-O|>|L-R| \Rightarrow\left\{\begin{array}{l}
S>O \Rightarrow O \\
S<O \Rightarrow S
\end{array}\right. \\
& |S-O|<|L-R| \Rightarrow\left\{\begin{array}{l}
L>R \Rightarrow R \\
L<R \Rightarrow L \\
U([L, R])
\end{array}\right.
\end{aligned}
$$

These rules compared the number of times a rat approached the same $(S)$ or opposite $(O)$ side as in an immediately preceding trial, in addition to the cumulative number of responses made to the left $(L)$ or right $(R)$ side. When the ratios of responses to same/opposite and left/right side were different, the relative offset of the poles (i.e., which was more posterior) was predetermined in the upcoming trial. When these ratios were equal, the poles were randomly arranged (with $U([L, R]$ being a distribution of equal left and right probabilities). These bias-correction rules were chosen to offset the occurrence of prevalent stereotypical trends, such as persistent responses to the same side or right-left-right switching between subsequent trials. Bias correction is common practice with rats (Carvell and Simons, 1990; Shuler et al., 2002) and monkeys (S. Barash, personal communication). We used four parameters computed across 10 consecutive trials, which made it difficult to predict the upcoming stimulus configuration.

Reward. Rewards consisted of diluted mango juice given in aliquots of $70 \mu \mathrm{l}$. The total amount of reward presented in each trial was adjusted according to the horizontal offset between the poles, i.e., the difficulty of the task. For offsets of $10-20,5-10,1-5$, and $\leq 1 \mathrm{~mm}$, one, two, three, and four aliquots, respectively, were presented. This inverse relationship between amount of reward and horizontal offset countered loss of motivation at increasingly difficult task levels.

Naive control. Besides testing rats with different whisker-array configurations, we also trained a group of naive control rats in the procedural, but not discriminative, parts of the task. After procedural training, these rats were trained in a random reinforcement schedule, whereby reward was guaranteed in every trial but reward location with respect to the poles 
was randomized. Thus, these rats did not associate the relative horizontal offset of the poles with the location of the reward. The behavior of this group served as a control for possible taskspecific behaviors exhibited by rats performing the localization task.

Tracking of head and whisker movements. Head and whisker movements during task performance were acquired with high-speed video (500 fps; MotionScope 8000PCI; Redlake) and tracked off-line. Our tracking method was described previously by Knutsen et al. (2005). Briefly, head positions were estimated by tracking reflections of two overhead light sources in the rat eyes. Locations and shapes of whiskers were estimated by fitting piecewise polynomials (splines) to the horizontal projections of whisker profiles in captured video frames. Whisker angles were computed at the whisker base (the most proximal point of the tracked whisker) from the coefficients of the most proximal polynomial of the whisker spline representation. For each training condition, head and whisker movements were tracked for a subset of all sessions. A particular whisker was easily located when a single row of whiskers was intact, because it could be identified directly by its posteroanterior location. Although whisker arcs are approximately orthogonal to the posteroanterior axis, the head typically tilted downward during task performance (Fig. 1C). Thus, the projected locations of whisker roots along an arc from a top view appeared separated as more ventral whiskers were shifted posterior relative to more dorsal whiskers. Although same-arc whiskers often overlapped in the video image, a particular whisker only had to be positively identified once and then tracked back or forth in the video through its continuous motion. When all whiskers were intact, individual whiskers could not be identified. However, we were still able to track individual whiskers within an intact whisker field through their continuous motion for entire trials.

Performance data of 616 sessions, which contained 39,718 trials, were analyzed. From this dataset, head and whisker movements were tracked in 2357 trials (3696 s of data). Two to four rats were tracked for each whisker-array condition, except for the ROWARC-FN condition (facial nerve-lesioned rats, whose whisker movements were negligible), in which only a single rat was tracked. On average, movements in 214 trials (336 s) were tracked for each whisker-array condition.

Facial nerve lesions. The buccal branch of the facial nerve, which supplies the efferents of intrinsic and extrinsic mystacial pad musculature, was lesioned bilaterally in three rats. These rats were lesioned after being successfully trained in the localization task with either a single row $(n=$ $1)$ or a single arc $(n=2)$ of whiskers intact. Before surgery, behavioral testing was stopped, and the rats were given food and water ad libitum for 5-7 d. Rats were anesthetized with pentobarbital (40 mg/kg, i.p.) and received an injection of atropine $(0.3 \mathrm{mg} / \mathrm{kg}$, i.m.) to prevent respiratory complications. Fur was shaved, and the facial nerve was exposed where the ramus buccolabialis superior and ramus buccolabialis inferior branches join ( $\sim 3-4 \mathrm{~mm}$ posterior to whisker E1). Approximately 1-2 $\mathrm{mm}$ of the nerve was removed with a pair of microdissecting scissors, and each cut end was briefly heated with a cauterizer to minimize nerve regrowth. The wound was sealed with cyanoacrylate gel (Loctite, Super Glue-3). Rats recovered for $5 \mathrm{~d}$ with ad libitum food and water, during which antibiotics (penicillin-streptomycin subcutaneously) and analgesics (5\% carprofen; Rimadyl, $5 \mathrm{mg} / \mathrm{kg}$, s.c.) were administered. The water-restriction schedule was reinstated $7 \mathrm{~d}$ after surgery, and behavioral testing was reinstated $1 \mathrm{~d}$ later.
B

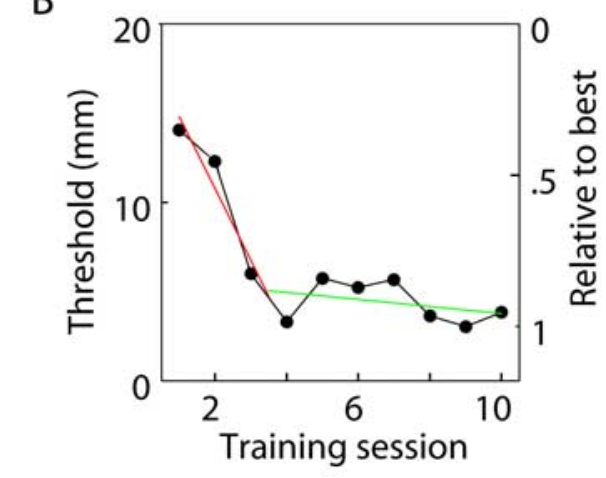

Training session

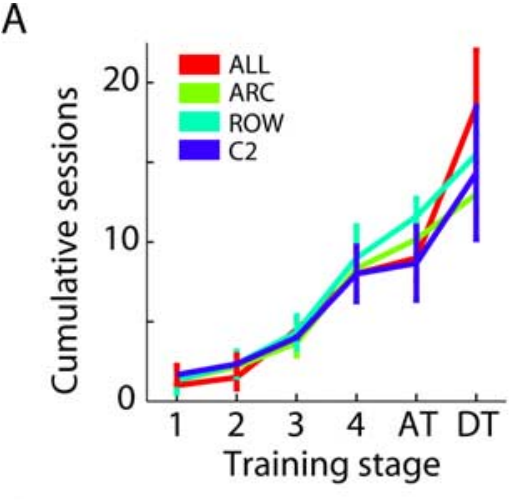

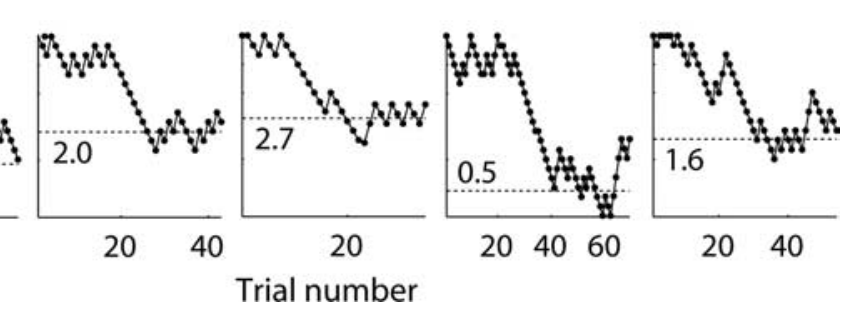

Trial number

Figure 2. Procedural and discriminative learning. $A$, Cumulative number of training sessions required for rats to successfully Dotted lines (and the numbers next to them) indicate the estimated threshold $\left(T_{e}\right)$ for the same session.

Psychophysical thresholds. We used an adaptive staircase procedure to estimate empirical localization thresholds $\left(T_{e}\right)$. Representative sequences of presented offsets ("staircases") of individual sessions are shown in Figure $2 C$. The staircase descends after a correct trial and ascends after an incorrect trial. The staircase should settle at a stable level if the performance of a rat is close to its optimal, although the descent through offsets $\geq T_{e}$ may be interrupted by errors. Therefore, we estimated $T_{e}$ as the minimum offset of the staircase after smoothing with a 15 -trial-wide boxcar filter.

To determine whether a distribution of $T_{e}$ for a given whisker configuration could have occurred if performance was random in every trial, we simulated a large number of staircases in which offsets were presented according to the same rules as in the actual experiment and the behavioral decision in every trial was random. We generated 5000 such staircases for each experimental session, with the same number of trials as in the experiment. A simulated threshold $\left(T_{s}\right)$ was estimated for each simulated staircase. The probability that the distributions of $T_{e}$ and $T_{s}$ obtained for individual whisker configurations were different were determined using the two-sample Kolmogorov-Smirnov goodness-of-fit hypothesis test.

Assuming that performance was random in all trials, we also estimated the probability $q$ that a particular $T_{e}$ could have been reached by chance in any one of all $n$ experimental sessions performed by a particular rat, as follows [Rice (1995), their page 100]:

$$
q=1-(1-p)^{n}
$$

where $p$ is the proportion of simulated staircases in which $T_{s} \leq T_{e}$.

To discriminate between rats that learned the task and those that did not, we defined a criterion performance level. We chose the learning criterion as having reached a threshold of $\leq m \mathrm{~mm}$ in two of three consecutive sessions. If the chance probability of obtaining a threshold below 

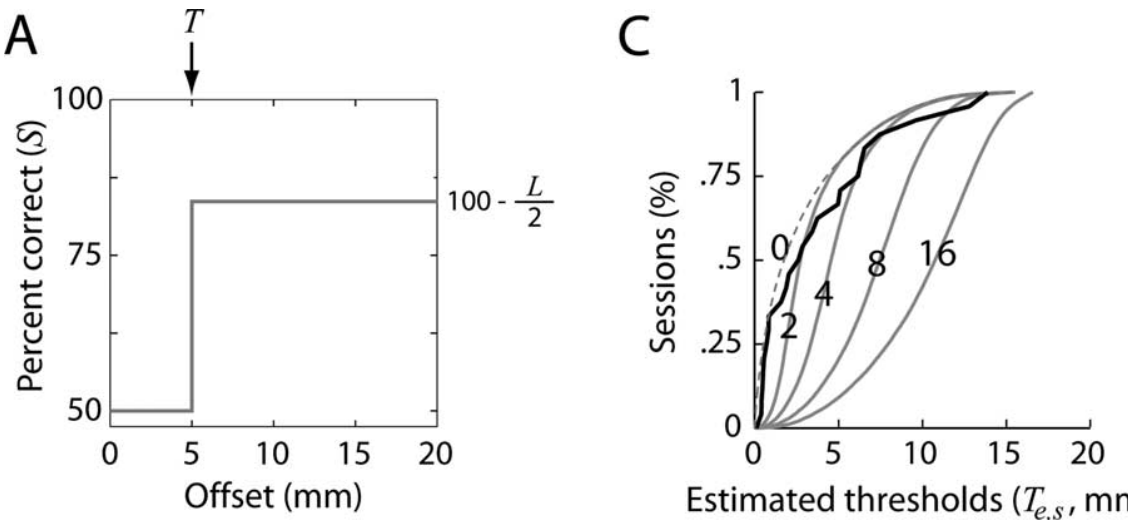

Estimated thresholds $\left(T_{e, s}, \mathrm{~mm}\right)$

B

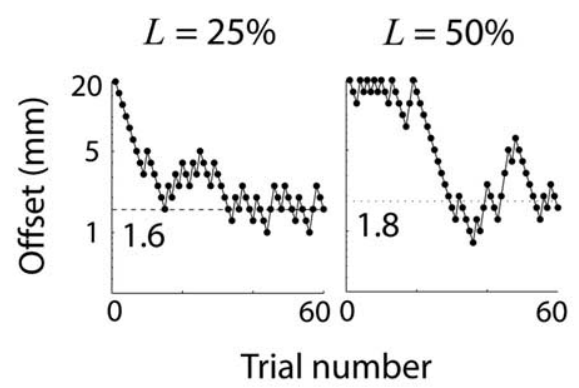

Figure 3. Performance model. $\boldsymbol{A}$, Localization performance was modeled with a simple approximation of an observer with a particular sensory threshold $(T)$ and a fixed rate of lapsing ( $L$ ). See Materials and Methods, Psychophysical thresholds. $\boldsymbol{B}$, Typical staircases generated by the performance model in $A$ with different values for $L$ ( 25 or $50 \%)$. The dotted line and number indicate the threshold estimated from the smoothed, simulated staircase $\left(T_{s} ; 15\right.$ bin boxcar filter). $C, I_{s}$ was estimated from staircases such as those in $\boldsymbol{B}$ generated by the performance model in $\boldsymbol{A}$. By repeating the simulation for a given combination of $T$ and $L$, a distribution of $T_{s}$ could be drawn. Gray lines depict CDFs of $T_{s}$ generated by the model when $T$ was $0,2,4,8$, or $16 \mathrm{~mm}$ and $L$ was $65 \%$. Solid gray lines indicate CDFs of $T_{e}$ given different values of $T$ that were significantly larger than the CDF of $T_{e}$ (black line; $p<$ 0.05). The dashed line indicates a CDF that was not significantly larger than the CDF of $T_{e}$. Numbers indicate $T$ for each CDF. D, The values of $T$ that generated distributions of $T_{s}>T_{e}$ depended on $L$; larger $L$ generated higher values for $T_{e}$ for a particular value of $T$. The shaded region above the curve includes combinations of $T$ and $L$ that yielded distributions of $T_{s}>T_{e}(p<0.05)$. Given the value of $L_{e}$ obtained empirically, the lowest $T$ in the shaded area was used as an approximation of the real sensory threshold (arrow).

$m$ in any given session was $p$ (as in Eq. 3 above), the probability of reaching criterion performance was as follows:

$$
P_{\text {criterion }}=\sum_{k=2}^{3} C_{3}^{k} p^{k}(1-p)^{3-k}
$$

where $C_{3}^{k}$ is the binomial coefficient. We selected a criterion performance $m=5 \mathrm{~mm}$, which had a chance occurrence of $<1 \%\left(p_{m=5}=0.0075\right)$.

The single-session $T_{e}$ could have been obtained by chance, and its probability of chance occurrence could have been estimated as in Equation 3. Alternatively, $T_{e}$ might have been achieved when performance was not random in all trials but rather reflected a "real" sensory threshold $(T)$ plus random behavior. For instance, a given $T_{e}$ might have resulted if $T>$ $T_{e}$ and the success rate at offsets $<T$ was higher than $67 \%$ by chance. Thus, it is necessary to estimate the minimal $T$ for which a given $T_{e}$ could have been obtained by chance (Fig. 3 ).

Localization performance was modeled by a simple approximation of an observer with a particular $T$ and a fixed rate of lapsing $(L)$. For all presented offsets $\leq T$, performance was always assumed to be based on guessing (i.e., $50 \%$ correct). When the offset was $>T$, the behavioral outcome was assumed to be correct in $100-L / 2$ percent of all trials, where $L$ is the probability of a lapse. We defined a lapse as the occurrence of a guess $(50 \%)$ when the offset was $>T$. This model is illustrated by its psychometric function in Figure $3 A$. In reality, $T_{e}$ may be influenced by noise, thus producing a sigmoidal or exponential psychometric function. However, for simplicity and clarity, we modeled $T$ as a step function, which allowed us to accurately estimate which $T$ would generate a distribution of $T_{s}>T_{e}(p<0.05$, Kolmogorov-Smirnov test), where $T_{s}$ are thresholds estimated from the simulated staircases.

We simulated staircases using the same rules as applied in the actual experiments (for examples, see Fig. $3 B$ ). Staircases generated using a lapsing rate of $0 \%$ produced errors in $50 \%$ of trials in which the offset was $\leq T$ and correct responses in $100 \%$ of trials in which offsets were $>T$. However, such staircases differed markedly from those obtained experimentally, because performance above $T_{e}$ in an experimental staircase was never $100 \%$ correct (see examples in Fig. 2C). Given $L$, it followed that the success rate $(S)$ was as follows:

$$
S=100-L / 2 \text { for offsets }>T .
$$

Thus, the experimental rate of lapsing was

$$
\begin{gathered}
L_{e}=200-2 S \text { when } S_{e} \geq 50, \\
L_{e}=100 \text { when } S_{e}<50,
\end{gathered}
$$

where $S_{e}$ is the experimental success rate. When our simulation model incorporated lapsing, it produced staircases very similar to those obtained experimentally (compare Figs. $2 C, 3 B$ ). For a particular combination of $T$ and $L$, we simulated 5000 such staircases, from which a distribution of $T_{s}$ could be produced. One such distribution was produced for each $T$ in the range of $0-20 \mathrm{~mm}$ (Fig. $3 C$ ), where $L$ was equal to the median $L_{e}$ of an individual whisker-array condition (Eq. 6). Each of these simulated distributions of $T_{s}$ were compared against the cumulative distributions of $T_{e}$ (KolmogorovSmirnov test) (Fig. 3D). We refer to the minimal $T$ that generated a distribution $T_{s}>T_{e}$ $(p<0.05$ ) (Fig. 3C,D) as the upper bound of the real sensory threshold.

Performance analysis. An example of a learning curve (threshold vs training session) is shown in Figure 2 B. Our performance analysis consisted of two phases. The initial phase was characterized by rapid (transient) learning, followed by a later phase with fluctuating performance. The end of the transient learning phase was defined ad hoc as the session in which a rat reached at least $80 \%$ of its best threshold (abbreviated as S-80). For individual rats, the percentage of the best performance was computed as $\left(20-T_{e}\right) /\left(20-T_{e-\text { min }}\right)$, where $T_{e \_ \text {min }}$ is the lowest measured threshold of the rat, and 20 is the largest presented offset.

For each learning curve, two linear regressions were fitted to thresholds of sessions: one before and one after S-80. Each regression coefficient was compared against a distribution of simulated regression lines obtained from 10,000 shuffles of the order of thresholds. This distribution represented the expected distribution of regressions if there was no relationship between performance and session number. Thus, the probability of each learning coefficient is $\left(R_{\text {sim }}>R\right) / 10,000$, where $\left(R_{\text {sim }}>\mathrm{R}\right)$ is the number of simulated coefficients greater than the learning coefficient.

Power spectral density estimates. Power spectral density (PSD) estimates of whisking movements were estimated using Welch's averaged, modified periodogram method as implemented in Matlab (release 14; MathWorks, Natick, MA). PSDs were computed for both whisker angle and whisker angular velocity. 
Statistics. Unless otherwise stated, all estimates of distribution centers are arithmetic means \pm SEM. When the median is cited, the spread indicates the standard error of the median $(1.25 \times$ SEM $)$. Except when noted, comparisons between distributions were based on the nonparametric Wilcoxon's rank sum test for equal medians and the Kolmogorov-Smirnov test. Cumulative distribution functions (CDFs) of thresholds in Figure 9 were fitted with a Weibull function, and the $R^{2}$ value of the exponential regression in Figure 12 estimated as follows:

$$
R^{2}=1-\frac{S S_{\text {Residuals }}}{S S_{\text {Total }}}
$$

where SS is the sum of squares of the residuals or thresholds (after subtracting the mean).

\section{Results \\ Procedural training}

Of the 34 rats that entered training, 26 (77\%) successfully completed the procedural and discriminative training. The remaining eight rats either did not complete the procedural training stages or became passive once introduced to discrimination trials. Training of these eight rats was discontinued when there was no sign of improvement within 5-10 training sessions (Table 1). For all conditions, procedural training was completed on average within seven to nine sessions (Fig. $2 \mathrm{~A}$ ). There were no significant differences between how fast rats with different whisker configurations acquired the procedural parts of the task ( $p>0.05, t$ test).

\section{Discriminative learning}

Procedural training (see Materials and Methods) was followed by a gradual introduction to discrimination trials (Fig. $2 \mathrm{~A}$, stage AT). Performance thresholds were estimated only for sessions run with $100 \%$ discrimination trials. An example of a learning curve is depicted in Figure $2 \mathrm{~B}$. We observed that most learning curves could be characterized by an initial, transient phase that lasted a few sessions and during which thresholds improved. After this transient phase, thresholds did not improve consistently. The number of discrimination trials per session was $63 \pm 19$ (mean $\pm \mathrm{SD})$.

During discriminative training, rats were presented with a variable horizontal offset between two vertical poles (Fig. 1). During pilot experiments $(n=6)$, performance rarely exceeded chance levels when both poles roved between randomly chosen locations across trials. Thus, the location of one of the poles (the posterior pole, or pedestal) was always fixed at the same horizontal distance from the front opening of the behavioral box (see Materials and Methods). From the onset of training, rats were trained with all, one row, one arc, or a single whisker intact on each cheek (conditions henceforth referred to as ALL, ROW, $\mathrm{ARC}$, and C2, respectively). With these conditions, the transient learning phase lasted $4 \pm 1.4$ training sessions (mean $\pm \mathrm{SD}$ ) (Table 1). This duration is a lower bound on the duration of the learning phase, because learning may also have occurred during sessions that included association trials. After the transient learning phase, performance for most rats still fluctuated between sessions. The SD of thresholds expressed in percentage of the best performance was $31.5 \pm 9.7 \%$ (mean \pm SD) during the learning phase and decreased to $21.8 \pm 2.4 \%$ after. During learning, this variability is attributable to session-to-session improvement in performance, as indicated by high and significant correlations between threshold and session number $\left(R_{\text {pre }}\right.$ and $\left.P_{\text {pre }}\right)$ (Table 1$)$. After learning, no consistent improvement was found in performance over time, as indicated by low and insignificant correlations $\left(R_{\text {post }}\right.$ and $\left.P_{\text {post }}\right)$.
We defined a performance criterion to distinguish between sessions with task-related performance and potentially random behavior. Our criterion that learning had occurred was that a threshold of $5 \mathrm{~mm}$ or less was reached in two of three consecutive sessions. The probability that this performance could occur by chance was 0.0075 (see Materials and Methods, Psychophysical thresholds). Of all rats $(n=20)$ initially trained with multiple whiskers intact (ALL, ROW, or ARC), 17 (85\%) reached criterion performance. Of rats $(n=6)$ initially trained with only a single whisker (C2), only one (17\%) reached criterion performance. Rats that reached criterion performance were eventually advanced to a new testing condition (ALL-ARC, ALL-C2, ROW$\mathrm{C} 2$, or ARC-C2) by trimming selected whiskers symmetrically on both sides of the face, leaving arc 2, row C, or the $\mathrm{C} 2$ whisker. Across the different groups, such trimming was done $2.7 \pm 0.8$ (mean $\pm \mathrm{SD}$ ) sessions after criterion performance was reached. These rats were then tested again with the localization task after 1-2 d. Three of four ALL-ARC rats were trimmed for a second time after criterion performance was reached with a single arc intact and then tested again with only a single whisker intact (ALL-ARC-C2). After reaching criterion performance in their initial training conditions, two rats with an arc (ARC) and one with a row (ROW) intact were retested with the same whisker array but after transecting the facial motor nerve (ROW-FN and ARC-FN). The various conditions tested are summarized in Table 1 .

After whisker trimming, performance typically did not change abruptly. Performance continued to vary considerably between sessions, with a consistent change in performance only becoming apparent when averaging across all sessions of a particular condition. The exceptions were ALL-ARC and ALL-C2 rats, whose thresholds abruptly increased after trimming and improved again within approximately three sessions (Table 1). In these two conditions, the largest number of whiskers were removed in a single step ( $>23$ compared with 4 in the other conditions; out of the 27 caudalmost whiskers, rows A to E, arcs 1-5, plus straddlers). As in the initial conditions, performance after the transient learning phase of rats in all whisker-trimmed groups fluctuated between sessions (12-27\%), but without any consistent improvement or decrement in performance with time.

\section{Head and whisker movement trends}

During pilot experiments without a nose poke, rats often rotated their heads up, down, or sideways during task performance and regularly came into contact with the vertical poles with their snouts. When the nose poke was introduced, rats approached the nose poke in a stereotypic manner and head movements to the sides were small. Representative head and whisker trajectories during localization trials are shown in Figure 4 (supplemental movies, available at www.jneurosci.org as supplemental material). Head movements consisted of three distinct phases: approach, discrimination, and withdrawal. Head movement trajectories of three rats were quantified (Fig. 5). During approach, the head exhibited low translational velocity (Fig. 5A), and the whiskers infrequently contacted the poles (Fig. $5 B$ ). In a brief discrimination period $(\sim 0.5 \mathrm{~s})$, the head moved forward and contacted the nose poke, whereas the probability of whiskers contacting the poles increased. After contact with the nose poke, the head withdrew rapidly. Throughout trials, the head remained clear of the poles. We found that the head was sufficiently close for the microvibrissas to contact one of the poles in $27 \%$ of randomly selected trials (no difference between whisker configurations). However, the macro-vibrissas contacted at least one of the poles in $96 \%$ of 
A
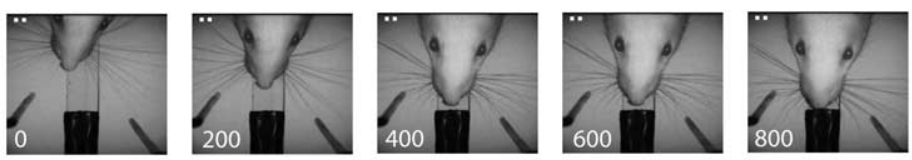

B
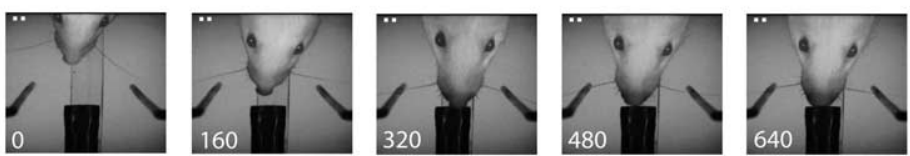

C
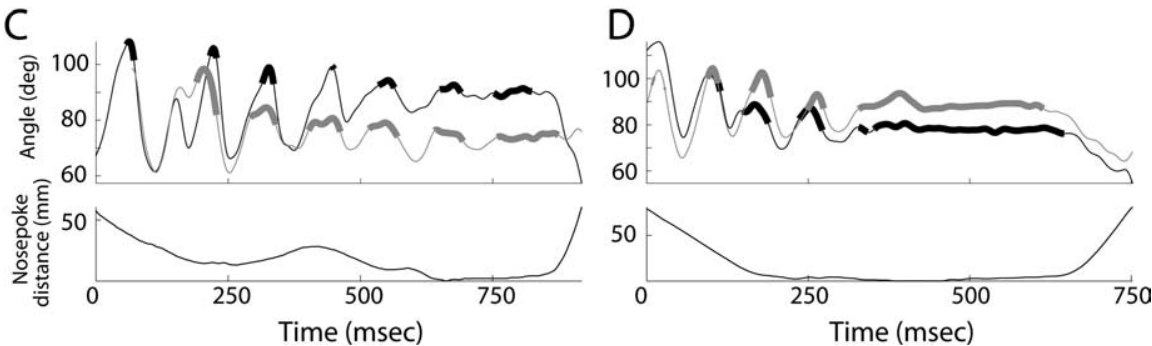

Figure 4. Head and whisker movements during task performance. $\boldsymbol{A}, \boldsymbol{B}$, Two examples of head and whisker movements during object localization trials. Each row depicts a single trial and shows six frames from the moment the rat entered the discrimination area, until it left. $\boldsymbol{A}, \mathrm{A}$ rat with all whiskers intact (the ALL condition). $\boldsymbol{B}, \mathrm{A}$ different rat with a single whisker intact on each side (ALL-C2). Because the trial durations were different for these two trials, intervals between still frames are different. The scale bar applies to all frames. C, An example of whisking behavior during object localization of a single-whisker rat, initially trained with all whiskers intact (ALL-C2). D, Example of whisking behavior of an ALL-ARC-C2 rat. In both $\boldsymbol{C}$ and $\boldsymbol{D}$, the $\mathrm{C} 2$ whisker on both sides (gray and black lines) was tracked from when the head entered the video frame and until it left. Top panels show the time-varying angle of the whiskers, relative to the head. Thick angle traces indicate moments when a whisker contacted one of the poles. The bottom panels show the time-varying distance of the nose from the nose poke.

A

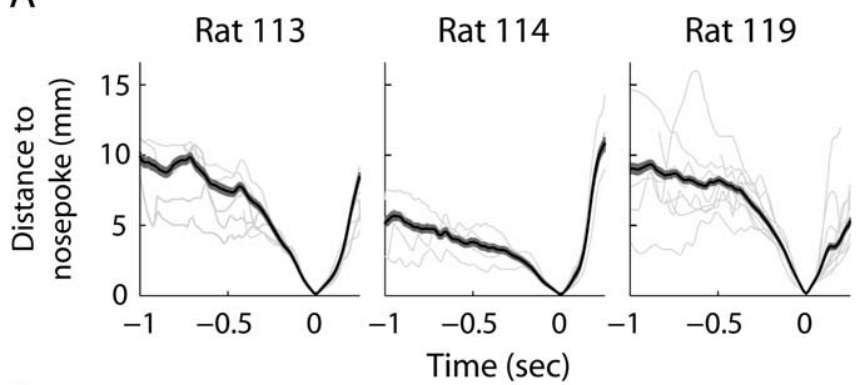

B

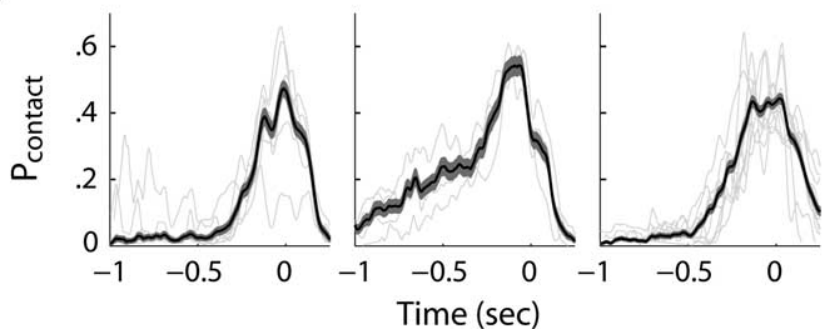

Figure 5. Head movements and whisker-object interactions during approach, discrimination, and withdrawal. $\boldsymbol{A}$, Average distance of the nose from the target nose poke during localization trials of three rats with a single pair of whiskers intact. Time 0 is the moment a rat contacted the nose poke. $\boldsymbol{B}$, The proportion of trials in which any whisker contacted an object, computed for each moment throughout trials. In $\boldsymbol{A}$ and $\boldsymbol{B}$, thin lines indicate session averages, and thick lines indicate the averages (and SEMs) of all trials across all tracked sessions. $C$, Probability of whiskers contacting the posterior (pedestal) pole when a single arc of whiskers was intact (ARC and ALL-ARC). D, Probability of contacting the pedestal when a single row of whiskers was intact (ROW). $\boldsymbol{E}$, Probability of contacting the pedestal when only a single whisker was intact on either cheek (ALL-ARC-C2,ALL-C2,ARC $-C 2$, ROW-C2, and (2).

the trials. The $\mathrm{C} 2$ whisker contacted the pedestal (posterior pole) with near equal probability across all whisker conditions (Fig. $5 C-E$ ). In the ARC and ALL-ARC groups, all whiskers along arc 2 contacted the pedestal with equal probability, whereas in the ROW condition, $\mathrm{C} 1$ contacted the pedestal in only $32 \%$ of trials
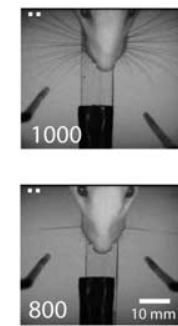

C

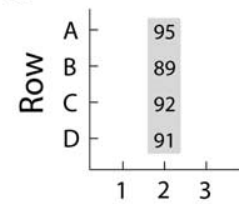

D

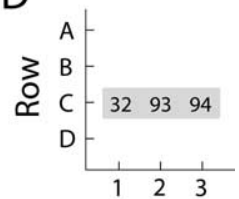

$\mathrm{E}$

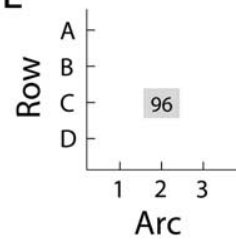

versus $93-94 \%$ in the case of whiskers C2 and C3. Altogether, these findings suggest that localization was mediated primarily by contact of the object with the macrovibrissas rather than with the microvibrissas, inter-vibrissal fur, or skin. The mode of contact was similar in the majority of trials: on contact, the whiskers rarely slipped vertically (along the length of pole) or radially (along the length of the whisker).

\section{Head movements and encoding of object location}

We presented rats with a variable horizontal offset in coordinates relative to the behavioral box. The nose poke, in addition to suppressing the tendency of rats to contact the poles with their snout, also ensured that the posteroanterior axis of the head was approximately aligned to the horizontal movement axis of the poles. Although head movements were small, they could still have been used along with whisker movements to improve localization of the poles. If the poles had no horizontal offset, any rotation of the head around the horizontal axis would have caused a nonzero horizontal offset in head-centered coordinates. We quantified such effects of head movements on relative object location by comparing the actual differences in whisker angle between left and right side with angles that should have resulted if the whiskers had zero curvature and the head was at and perpendicular to the nose poke (Fig. 6). Differences in whisker angle between the two sides during contact (Fig. $4 C, D)$ may encode the relative horizontal offset of the poles; in fact, primary sensory neurons have been found whose activity carry information about protraction angle or phase (Szwed et al., 2003). At 1 and 5 $\mathrm{mm}$ horizontal offset, the angular difference was $\sim 2.5$ and $9^{\circ}$, respectively. For horizontal offsets $>3 \mathrm{~mm}$, actual angular differences were significantly smaller than predicted with a stationary head coaligned to the horizontal axis of the poles (Fig. $6 A)$. This deviation could be explained if head orientation was biased toward the pedestal (posterior). Indeed, rats increasingly oriented their head toward the posterior pole with increasing offsets (Fig. $6 B)$. Whereas head-rotation bias was negligible for small offsets, it increased to $\sim 4^{\circ}$ toward the posterior pole at the largest (20 $\mathrm{mm})$ offsets.

Rats can discriminate radial apertures that differ in widths as little as $3 \mathrm{~mm}$ (Krupa et al., 2001). The current task does not attempt to establish perfect control of object location relative to the head, and head movements could therefore have induced radial cues that in turn signaled relative horizontal location. In- 
deed, the radial point of contact along the whisker shaft with the posterior pole decreased slightly with increasing horizontal offsets (Fig. 6C), consistent with the observation that the head oriented toward the posterior pole when horizontal offsets were large (Fig. $6 \mathrm{~B}$ ). For horizontal offsets $>7 \mathrm{~mm}$, the difference in the radial point of contact along the whisker shaft on anterior and posterior side was $>3 \mathrm{~mm}$. However, when horizontal offsets were $<7 \mathrm{~mm}$, the radial difference was below the $3 \mathrm{~mm}$ radial discrimination threshold (Krupa et al., 2001). Thus, we conclude that the radial cue is not a major factor in the encoding of small horizontal offsets. This result did not differ significantly between rats from conditions before or after trimming of the whiskers.

Although the current task did not allow optimal control of stimulus presentation (animals were unrestrained but stimuli were fixed in location), rats developed stereotyped behaviors characterized by distinct approach, discrimination, and withdrawal phases (Fig. 5). As horizontal offsets were reduced, rats increasingly aligned their heads parallel to the axis of the horizontal offset between the poles. Thus, for small offsets, head movements minimally changed how angular and radial cues encoded small differences in horizontal object location compared with if the head was fixed in place (Fig. 6).

\section{Spatial sampling}

Performance in a variety of tasks (tactile, visual, and auditory) can be improved by using a fixed reference signal, also referred to as a pedestal (Green and Swets, 1966). Here, we provided a pedestal by positioning the posterior pole at the same distance from the behavioral box. With a fixed reference, the localization task could be reduced to a detection task in which the pedestal is searched for on either one or both sides. We examined localization behavior to determine which task was actually performed, by assuming that a rat conditioned to detect the pedestal would only scan the region in the vicinity of the pedestal. The majority of rats, however, scanned the entire distance up to the location of the more anterior pole, even for the largest offsets. The distribution of the ratio of the scanned region between the pedestal (at $0 \mathrm{~mm}$ ) and the location of the more anterior pole $(>0 \mathrm{~mm})$ is shown in Figure 7. Across all whisker configurations and offsets, rats scanned $90 \pm 16 \%$ of the gap between the pedestal and the anterior pole. Also, after an initial contact and when no whiskers were contacting either pole, protraction amplitudes of whiskers on the same side as the anterior pole were $7.6 \pm 0.2^{\circ}$ larger than amplitudes of whiskers on the side of the pedestal (significant for all rats, $p<0.0001$ ) (Fig. $4 C$ ). In other words, rats were reaching to contact the anterior pole, suggesting that task performance was not based on detection of the pedestal itself but rather on a comparison between the location of the anterior pole and the pedestal.

\section{Discrimination speed}

The sampling phase was brief compared with the total trial duration (Fig. 5B). Typically, only a small number of discrete contacts were made between the whiskers and objects (Fig. $8 A$ ). Singleintervals.
B

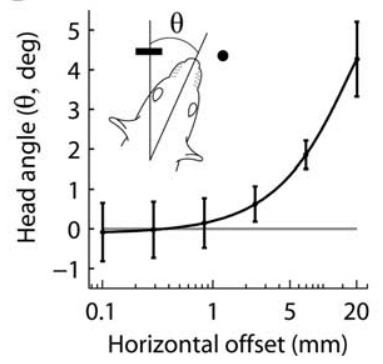

C

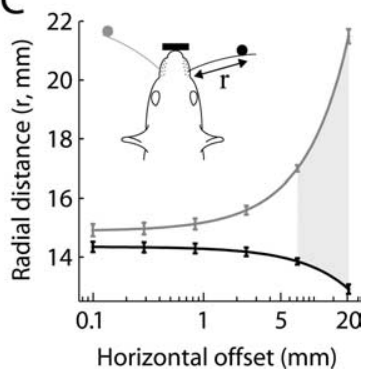

Figure 6. Encoding of object location. A, Horizontal object offset versus the absolute difference in whisker angle, relative to thick black bar indicate the nose poke. Black lines (plus gray in C) indicate the best quadratic fit to the data and the $95 \%$ confidence

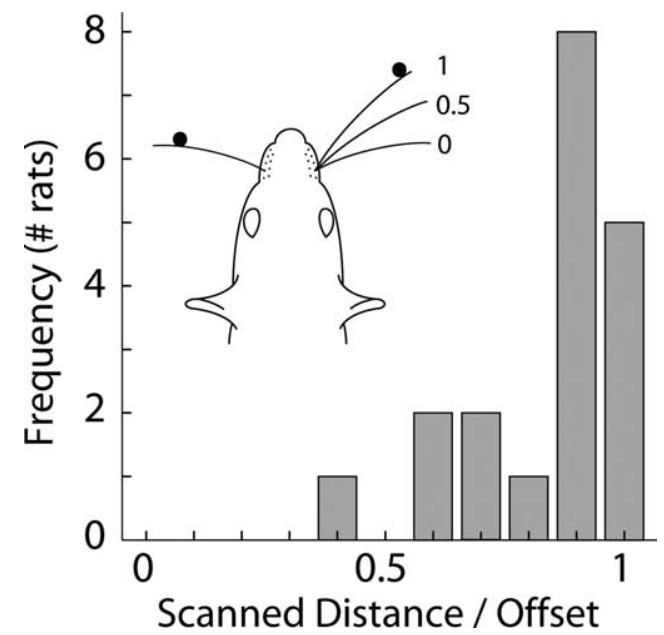

Figure 7. Horizontal region scanned between poles. A histogram of the ratio of the distance whiskers scanned beyond the location of the pedestal $(0 \mathrm{~mm})$ and the location of the anterior pole ( $>0 \mathrm{~mm}$; averages of different rats) is shown. An area was considered scanned if a whisker at any time moved through it during a trial. Thus, if the anterior pole was placed $20 \mathrm{~mm}$ behind the posterior pole and a whisker moved up to within $10 \mathrm{~mm}$ of the anterior pole, $50 \%$ of the horizontal gap between the poles was considered scanned ( 0.5 in the histogram). All tracked trials were included ( $n=19$ rats, 9 groups). Rats with all whiskers intact (ALL) were not included, because a significant proportion of the whiskers were not tracked.

whisker rats made 6-10 (interquartile range; median of 8) whisks during a trial (Fig. $8 \mathrm{~A}$, gray fill), and 3-6 (median of 4 ) discrete contacts with the poles (Fig. $8 A$, black bars). The number of contacts differed significantly between the sides of the anterior and posterior pole $(p<0.001)$ (Fig. $8 A$, inset). For horizontal offsets $>7.5 \mathrm{~mm}$, more contacts were made with the posterior (pedestal) than the anterior pole $(p<0.01)$. We found no difference between the numbers of contacts made with either pole during correct and incorrect trials. The cumulative contact time also differed significantly between the side of the posterior (median of $286 \mathrm{~ms}$ ) and anterior (median of $108 \mathrm{~ms})$ poles $(p<$ 0.001) (Fig. 8 B). Contact durations with the posterior (pedestal) pole did not change as horizontal offsets changed. However, con- 

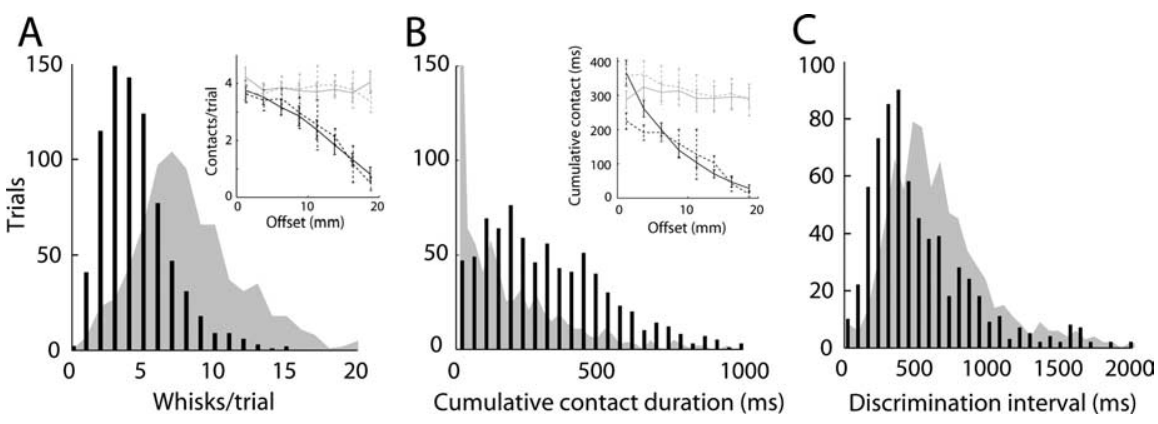

Figure 8. Whisk counts and discrimination speed. $\boldsymbol{A}$, Distribution of the number of contacting whisks (black bars) and contacting plus noncontacting whisks (gray area) during localization trials. The inset shows the number of whisks that contacted the anterior (black) and posterior (gray) poles during correct (solid lines) or incorrect (dashed lines) trials as a function of horizontal offset. $\boldsymbol{B}$, The distribution of cumulative contact durations with either the anterior (gray fill; median of $108 \mathrm{~ms}$ ) or the posterior (black bars; median of $286 \mathrm{~ms}$ ) pole is shown. The inset shows cumulative contact durations with anterior (black) and posterior (gray) poles as a function of horizontal offset separately for correct (solid lines) and incorrect (dashed lines) trials. C, Distributions of delays between the time of the first whisker- object contact and nose-poke detachment (black bars; median of $406 \mathrm{~ms}$ ) and of intervals between the first object contact and the last object detach in a trial (gray fill; median of $588 \mathrm{~ms}$ ). In $\boldsymbol{A}-\boldsymbol{C}$, data were included from sessions in which rats had a single pair of whiskers intact ( $A L L-C 2, A L L-A R C-C 2, A R C-C 2$, and ROW-C2) and in which the threshold of the session $(2.4 \pm 0.9 \mathrm{~mm}$ ) had a chance probability $\leq 0.05$ ( 9 rats, 18 sessions, 777 trials).
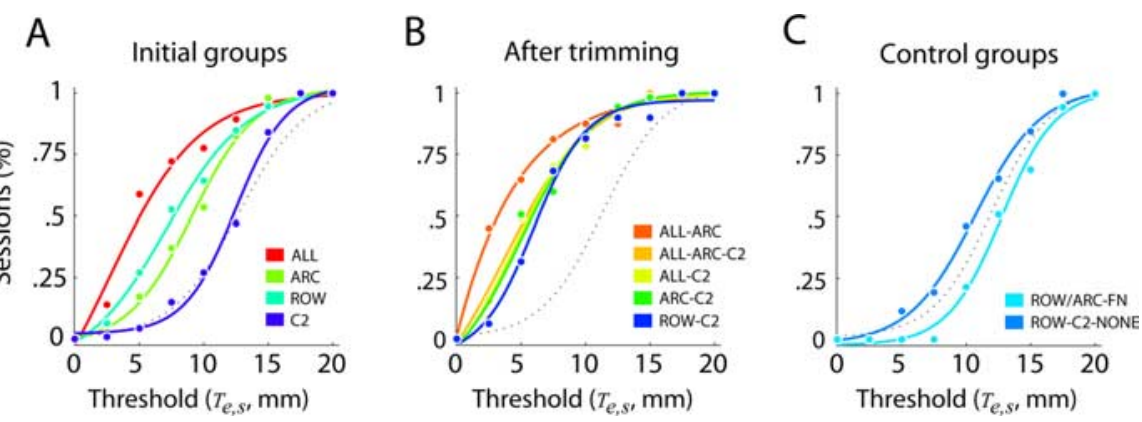

Figure 9. Distribution of performance thresholds. CDFs of $T_{e}$ for initial training conditions $(\boldsymbol{A})$, after whisker trimming $(\boldsymbol{B})$, and for rats with facial nerve lesions (C) (ROW/ARC-FN) and without any intact whiskers (ROW-C2-NONE). CDFs were binned at 2.5 $\mathrm{mm}$ intervals and fitted with a sigmoid Weibull function (solid lines; $R^{2}>0.98$ in all cases). Dotted lines indicate the CDFs of $T_{s}$ when performance was random at all offsets (for details, see Materials and Methods, Psychophysical thresholds and Fig. 3). All CDFs, except for those of C2, ROW/ARC-FN, and ROW-C2-NONE, were significantly greater than the simulated $\operatorname{CDF}(p<0.0001)$.

tact durations (and the number of contacts) reduced as offsets increased (i.e., the anterior pole was placed farther way) (Fig. $8 \mathrm{~B}$, inset). Again, we found no significant differences in cumulative contact duration between correct and incorrect trials. We inferred that the maximal duration of discrimination should be the time interval between the first and last whisker-object contact in a trial. During withdrawal, rats often (but not always) oriented their heads toward the side to which they would respond, which suggests that a decision was made soon after contacting the nose poke. Thus, an estimate of the minimal duration of discrimination is the interval between the first whisker-object contact and the moment of nose-poke contact. These two estimates of the discrimination speed were significantly different $(p<0.001)$ and had respective medians of $588 \mathrm{~ms}$ (contact-to-contact) (Fig. $8 \mathrm{C}$, gray fill) and $406 \mathrm{~ms}$ (contact-to-nose poke) (Fig. $8 \mathrm{C}$, black bars).

\section{Localization performance}

In all sessions, localization acuity was quantified by the threshold $T_{e}$, which was estimated from the minimum running average of the presented trial-to-trial offsets. Because offsets were reduced after correct trials and increased after incorrect ones, $T_{e}$ decreased with improving performance (see examples in Fig. $2 C$ ).

$T_{e}$ varied between rats tested with different whisker configu- rations (Figs. 9, 10). As noted above in Discriminative learning, rats reached criterion performance when initially trained with all (ALL), a row (ROW), or an arc (ARC) of whiskers intact. Although rats trained with a single pair of whiskers (C2) intact from the training onset failed to reach criterion performance, all rats initially trained with any combination of multiple whiskers and later retested with only a single pair of whiskers intact (ALLC2, ALL-ARC-C2, ARC-C2, and ROWC2) did reach criterion performance. CDFs of $T_{e}$ obtained in each of the initial training conditions and after subsequent whisker trimming are shown in Figure 9, $A$ and $B$. Dotted lines indicate CDFs of $T_{s}$ obtained by simulation of performance levels expected from entirely random behavior ( $50 \%$ correct at all offsets). For all conditions except C2 ( $p=0.59)$, ROW/ ARC-FN $(p=0.94)$ and ROW-C2NONE $(p=0.07)$, the CDFs of $T_{e}$ were shifted to the left of the chance CDF (i.e., offsets were smaller than expected by chance; Kolmogorov-Smirnov test, $p<$ $0.0001)$. After trimming to a single pair of intact whiskers, rat performance converged to similar performance levels, regardless of what the initial whisker configuration was (Fig. 9B). The average $T_{e}$ of each whisker configuration group is shown in Figure $10 B$. Rats initially trained with all whiskers intact (ALL) reached an average $T_{e}$ of $7.2 \pm 0.5 \mathrm{~mm}$, whereas rats with a single row (ROW) or arc (ARC) reached $8.7 \pm 0.6$ and $8.9 \pm 0.5 \mathrm{~mm}$, respectively. The overall performance of rats initially trained with a single pair of whiskers intact $(\mathrm{C} 2 ; 12.4 \pm 0.5 \mathrm{~mm})$ did not exceed chance levels. Trimming of multiple intact whiskers to yield a single pair of intact whiskers typically reduced (i.e., improved) $T_{e}$. Reducing the number of intact whiskers of ALL rats to a single pair (ALL-C2) lowered $T_{e}$ to $5.7 \pm 0.6 \mathrm{~mm}$, although this improvement in itself was not significant ( $p=0.16$, Wilcoxon's test). The improvement was larger, and significant, for rats initially trained with a row or an arc who, after trimming to yield a single pair of intact whiskers, reached thresholds of $5.4 \pm 0.7$ $\mathrm{mm}$ (ROW-C2; $p=0.05)$ and $4.2 \pm 0.7 \mathrm{~mm}(\mathrm{ARC}-\mathrm{C} 2 ; p=$ 0.002 ), respectively. Improvement was largest in ALL-ARC rats (initially trained with all and then with one arc of intact whiskers), with a reduction of $T_{e}$ from $7.2 \pm 0.5$ to $2.7 \pm 0.8 \mathrm{~mm}(p<$ $0.001)$. ALL-ARC rats continued to perform at an improved level when trimmed for a second time to yield only a single pair of intact whiskers (ALL-ARC-C2; $4.6 \pm 0.8 ; p<0.04$ ). However, $T_{e}$ after the second trimming was higher than after the first trimming (ALL-ARC; $p=0.031$ ). We were not able to consistently attribute improvements in performance after whisker removal to any additional training received. For all whisker configurations tested, we did not observe any consistent session-to-session improvement beyond the $\mathrm{S}-80$ session. Although animals with reduced sets of intact whiskers participated in a greater number of training sessions, we did not find any consistent correlation be- 
A

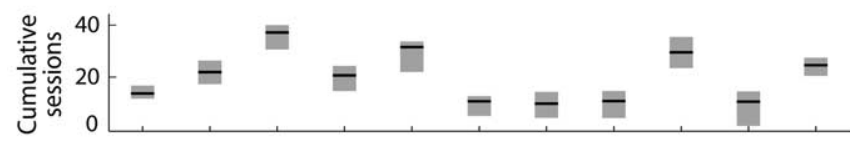

B

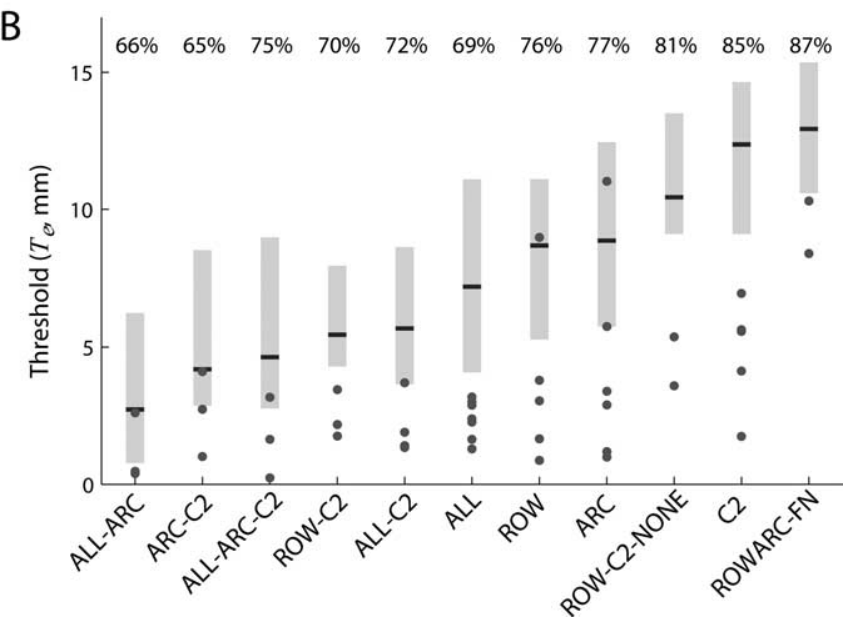

Figure 10. Horizontal localization performance. $A$, Cumulative number of training sessions for each whisker configuration. Black horizontal lines indicate the mean cumulative session number averaged. The gray boxes indicate the range of cumulative sessions in the different groups; lower and upper ends indicate the smallest and largest cumulative session, respectively, averaged across rats in that group. $\boldsymbol{B}$, Distributions of $T_{e}$ across all steady-state sessions of all rats for a given whisker configuration. Black lines indicate the median $T_{e^{\prime}}$ and the lower and upper end of the gray bars indicate the first and third quartiles, respectively. The lowest $T_{e}$ reached by each individual rat is indicated by for all whisker configurations. Numbers above bars indicate the median $L_{e}$ computed in sessions (see Fig. 3, Eq. 6). The range of these numbers $(65-87 \%)$ entails average success rates of $57-68 \%$ (Eq. 5). These low rates result from the staircase method of stimulus presentation with presentations close to sensory threshold $T$, in which near-chance performance is expected.

tween the total amount of training (cumulative session count) and performance across all whisker configurations (Fig. 10 A; Table 1).

Reliable horizontal localization was whisker mediated. Rats that initially succeeded in reaching criterion performance (ROW-C2), failed to reach criterion performance after all whiskers were removed (ROW-C2-NONE; $T_{e}=10.4 \pm 0.7 \mathrm{~mm}$ ). Reliable localization was also dependent on the ability to move the whiskers, because rats with permanent whisker paralysis (ROW/ARC-FN) failed to reach criterion performance $\left(T_{e}=\right.$ $12.7 \pm 0.8 \mathrm{~mm})$.

\section{Limits of horizontal acuity}

The average performance reached by rats does not indicate their limit of horizontal acuity. The lowest $T_{e}$ reached by individual rats are shown in Figure $10 B$ (gray dots). In the condition with lowest average $T_{e}$ (ALL-ARC), the lowest individual $T_{e}$ was $0.4 \pm$ $0.5 \mathrm{~mm}$. The lowest $T_{e}$ of individual rats in all groups was 0.24 $\mathrm{mm}$ (in ALL-ARC-C2). Assuming random performance in all trials $(L=100 \%)$, the probability of such a threshold occurring in a simulated session was only 0.0006 (Eq. 3). However, the probability of this threshold occurring by chance increases significantly if the behavioral outcome is not random in all trials but rather is limited by a given sensory threshold $(T)$. To obtain better approximations of real sensory thresholds, we compared experimental thresholds $\left(T_{e}\right)$ with simulated thresholds $\left(T_{s}\right)$ obtained using different values of $T$ in a Monte Carlo simulation (see Materials and Methods, Psychophysical thresholds). The
Table 2. Localization acuity across groups

\begin{tabular}{llll}
\hline & & \multicolumn{2}{c}{ Frequency of $T_{e}<4.8 \mathrm{~mm}$} \\
\cline { 3 - 4 } Group & $T_{\text {max }}(\mathrm{mm})$ & Rats, $\%(n)$ & Sessions, $\%(n)^{a}$ \\
\hline ALL & 7.1 & $38 \%(3)$ & $27 \%(7)$ \\
ALL-C2 & 6.3 & $75 \%(3)$ & $11 \%(7)$ \\
ALL-ARC & 1.5 & $75 \%(3)$ & $32 \%(12)$ \\
ALL-ARC-C2 & 2.8 & $67 \%(2)$ & $16 \%(5)$ \\
ROW & 8.7 & $17 \%(1)$ & $7 \%(1)$ \\
ROW-C2 & 6.5 & $0 \%(0)$ & \\
ROW-C2-NONE & $b$ & $0 \%(0)$ & $9 \%(3)$ \\
ARC & 9.8 & $33 \%(2)$ & $17 \%(4)$ \\
ARC-C2 & 4.9 & $33 \%(1)$ & \\
ARC/ROW-FN & $b$ & $0 \%(0)$ & \\
C2 & $b$ & $0 \%(0)$ & \\
\hline
\end{tabular}

${ }^{a}$ Includes only sessions of rats that reached threshold $<4.8 \mathrm{~mm}$ in one session or more. Thus, no result is obtained in groups in which none of the rats reached this level of performance.

${ }^{b}$ No significant $T_{\max }$ was found below $20 \mathrm{~mm}$

minimal $T$ that generated a distribution $T_{s}>T_{e}($ with $p=0.05)$ is given here as an approximation of the upper bound of the real sensory threshold.

Upper bounds of the real sensory thresholds in the groups in which rats reached criterion performance were in the range of $1.5-9.8 \mathrm{~mm}$. These values followed the general trend of the average $T_{e}$ obtained in individual groups. The upper bounds of real thresholds (denoted $T_{\max }$ ) were higher for the initial training conditions and lower for whisker configurations after trimming (Table 2). Lowest thresholds were reached by the ALL-ARC group (upper bound of $1.5 \mathrm{~mm}$ ) and the ALL-ARC-C2 group (upper bound of $2.8 \mathrm{~mm}$ ).

\section{Relationship between horizontal acuity and whisker spacing}

The horizontal location of an object could be encoded by the combination of contacting whiskers. For example, the identities of contacting whiskers $(\mathrm{C} 1, \mathrm{C} 2$, etc.) signal the relative horizontal location of an object, because objects placed increasingly anterior are less likely to be contacted by the most posterior whiskers. Similarly, the total number of contacting whiskers may also signal horizontal location, because fewer whiskers will reach and contact increasingly anterior objects. Common to such spatial codes is that, in the absence of whisker movements, their resolution is limited by the spacing between neighboring whiskers. For example, if only $\mathrm{C} 3$ contacts an object $\mathrm{A}$ and both $\mathrm{C} 2$ and $\mathrm{C} 3$ contact an object $\mathrm{B}$, then $\mathrm{B}$ should be posterior to A. If the horizontal offset between $A$ and $B$ is less than the spacing between $C 2$ and $C 3$, however, both objects may be contacted by C3 only. Such a spatial cue will therefore not suffice to discriminate horizontal offsets smaller than the spacing of neighboring whiskers.

We compared performance levels with the actual whisker separations measured during task performance (Fig. 11). Close to the whisker base, distances between neighboring whiskers of the same row were $1.8 \pm 0.1 \mathrm{~mm}$ for the whisker pair $\mathrm{C} 1-\mathrm{C} 2$ and $1.5 \pm 0.2 \mathrm{~mm}$ for $\mathrm{C} 2-\mathrm{C} 3$, similar to the distances according to Rice and Munger (1986). Distances between the whisker shafts were considerably larger, because whiskers diverge from each other as they exit the follicle (Brecht et al., 1997). The objects in our task were contacted $14.2 \pm 0.5 \mathrm{~mm}$ out on the whisker shaft (between 30 and $70 \%$ of whisker length, depending on whisker identity) (Fig. 11A). At this radial distance, the horizontal separation of neighboring whiskers of the same row $(\mathrm{C} 1-\mathrm{C} 2$ and $\mathrm{C} 2-$ C3) was $4.8 \pm 0.3 \mathrm{~mm}\left(21.3 \pm 0.8^{\circ}\right)$ (Fig. $\left.11 B\right)$.

Estimates of the upper bounds of real sensory thresholds obtained in the previous section were smaller than this inter- 
A

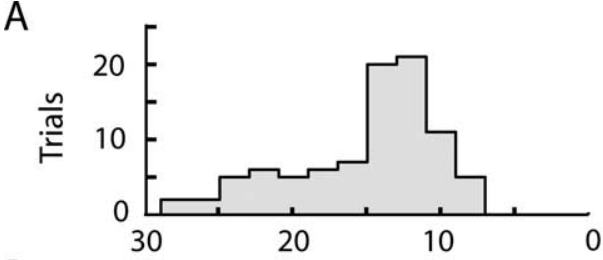

B

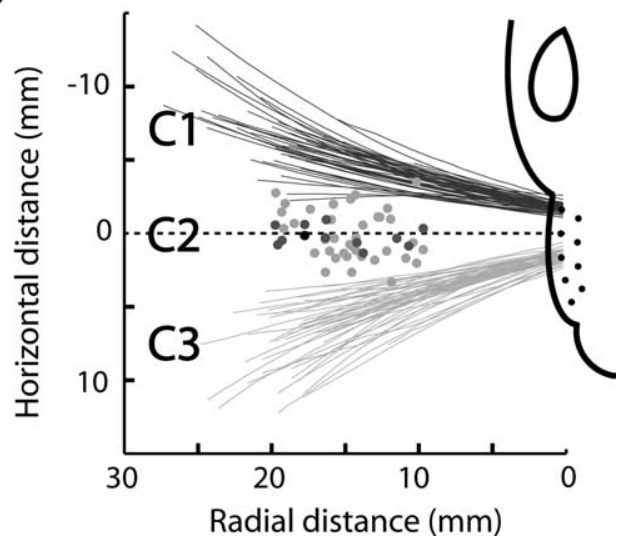

Figure 11. Separation distance of neighboring whiskers and discrimination thresholds. $\boldsymbol{A}$, Distribution of radial contact locations along the whisker shaft during object localization. $\boldsymbol{B}$, The spatial distance between neighboring whiskers. Horizontal separation, as a function of radial distance, of whisker pairs C1-C2 (dark gray) and C2-C3 (light gray), obtained while rats from the ROW configuration were whisking through the air. Each line is the average horizontal separation in a single trial. Dots indicate performance thresholds, selected from all whisker configurations, significantly smaller than the average inter-vibrissal distance (light gray, $p<$ 0.05 ; medium gray, $p<0.01$; dark gray, $p<0.001$ ). The location of the threshold values along the abscissa were randomized, because the radial contact data were lacking for the majority of sessions from which these were obtained. The rat head is drawn approximately to scale. Radial positions in $\boldsymbol{A}$ and whisker separations in $\boldsymbol{B}$ were gathered from 90 trials performed by two rats with the ROW whisker configuration.

vibrissal spacing in the ALL-ARC-C2 and ALL-ARC groups. In all other groups (ALL, ROW, ARC, ROW-C2, ALL-C2, and ARC-C2), they were larger than the inter-vibrissal spacing. We also estimated the probability that a particular $T_{e}$, measured in a single experimental session, could occur by chance by assuming that the real sensory threshold was the same as the inter-vibrissal distance. We repeated our simulation and obtained the probability of an individual $T_{e}$ being generated by chance, assuming $T$ was $4.8 \mathrm{~mm}$ (Eq. 3). Rats reached thresholds below the inter-vibrissal distances in the groups ALL, ALL-ARC, ALL-ARC-C2, ALL-C2, ARC, ARC-C2, and ROW $(p<0.05)$. The number of rats that reached this level of performance and the frequency at which it occurred are shown in Table 2. In the groups C2, ROW-C2, ROW-C2-NONE, and ROW/ARC-FN, none of the rats reached thresholds lower than the inter-vibrissal separation.

\section{Relationship between whisking and acuity}

During horizontal object localization, rats always moved their whiskers, unlike the behavior of rats that have been trained to discriminate radial apertures (Krupa et al., 2001). Furthermore, although rats unable to move their whiskers because of motor nerve lesions are able to perform radial discriminations (Krupa et al., 2001), vibrissal paralysis caused performance in our horizontal object localization task to drop to chance levels $(p<0.001)$ (Figs. 9, 10B). Thus, horizontal localization was not possible without active whisker movement.

During horizontal localization trials, rats made several movements (whisks), which brought the whiskers in contact with the vertical poles (Fig. 8). There was no correlation between the number of discrete whisks made in trials and acuity $\left(R^{2}=0.01\right)$ and no difference in total whisk count $(p=0.6)$ or discrimination duration $(p=0.46)$ between correct and incorrect trials. Regression analysis did not reveal any significant change in whisk count $\left(R^{2}=0.01\right)$ or discrimination duration $\left(R^{2}=0.03\right)$ as a function of offset magnitude (i.e., task difficulty). On average (across all groups except ALL), any individual whisker contacted the ipsilateral object $22.3 \pm 11.1 \%$ of the time a rat was in the discrimination area. There was no significant correlation between this percentage and acuity $\left(R^{2}=0.16\right)$.

Whisking behavior was further quantified by computing PSDs of the whisker angle and the angular velocity. Whereas PSDs of whisker angles often peaked at $0 \mathrm{~Hz}$ (Fig. 12A), PSDs of angular velocity peaked at the dominant whisking frequency of a trial (Fig. 12B). The PSDs of whisker angular velocity contained peaks at previously characterized whisking frequencies (Welker, 1964; Semba and Komisaruk, 1984; Carvell et al., 1991, 1996; Carvell and Simons, 1995; Nicolelis et al., 1995; Fee et al., 1997; Hamada et al., 1999; Gao et al., 2001; O’Connor et al., 2002; Berg and Kleinfeld, 2003b). In our task, the frequency of whisking was distributed across a wide range, with the majority (93\%) of dominant whisking frequencies occurring in the range of $5-20 \mathrm{~Hz}$ (Fig. 12C). For all whisker-array configurations, the distribution of whisking frequencies was bimodal, with a narrow peak centered at $8.3 \pm 1.3 \mathrm{~Hz}$ and a second, broader peak centered at 14 $\mathrm{Hz}$. We estimated the bandwidth of the $8.3 \mathrm{~Hz}$ peak at halfamplitude to be $2.2 \pm 0.6 \mathrm{~Hz}$, which is in agreement with Berg and Kleinfeld (2003a). Previously, the $8.3 \mathrm{~Hz}$ peak was associated with exploratory whisking behavior, and typically little additional whisking occurs at higher frequencies in rats not performing a contact-dependent task (Gao et al., 2001; Berg and Kleinfeld, 2003a). In contrast, during horizontal localization, whisking at frequencies above $10 \mathrm{~Hz}$ occurred in $49 \%$ of all trials. Thus, either the presence of objects or task-specific requirements appeared to increase whisking frequencies during object localization. During an object detection task, whisking frequencies are higher relative to whisking during an object discrimination task (Harvey et al., 2001). Thus, in those conditions, object contact in the absence of discrimination appeared to increase whisking frequencies.

During horizontal localization, there was no significant correlation between the distribution of whisking frequencies (e.g., shifts to higher or lower frequencies) and performance levels. However, there was a strong correlation $\left(R^{2}=0.92 / 0.84\right.$, exponential/linear fit; $p=0.001$ ) (Fig. 12D) between performance levels and the total amount of power (area underneath the PSD) in the $5-25 \mathrm{~Hz}$ range. This correlation persisted for any $10 \mathrm{~Hz}$ bin in the $5-25 \mathrm{~Hz}$ range and thus did not appear specific to any particular range of whisking frequencies. We did not find any correlation between performance and power in the nonwhisking range $(30-50 \mathrm{~Hz} ; p=0.18)$. The correlation between power and performance did not change if correct or incorrect trials were analyzed separately for the $5-25 \mathrm{~Hz}$ range.

The behavior and performance of rats trained with a single pair of whiskers intact from onset of training (C2) differed markedly from that of the other groups. Only one of the six C2 rats reached criterion performance, and the performance of the whole group was not significantly better than chance levels (Fig. 10). Nevertheless, the net whisking power of the rats in this group was significantly higher than seven of nine of the other groups $(p<$ 0.001 ) (Fig. $12 D)$, as was that of naive rats $(p<0.001)$ (Fig. 12D, arrow). The naive control group indicates the default behavior, without discrimination, in our task. Thus, one effect of the local- 
ization task appears to be initial reduction of the whisking spectral power. Conversely, and seemingly paradoxical, an increase in spectral power toward its naive "default" level correlated with an improvement in performance. With regard to the cause of the observed changes in spectral power, both whisking amplitudes $\left(R^{2}=0.61 ; p=0.02\right)$ and velocity $\left(R^{2}=\right.$ $0.79 ; p<0.001)$ increased significantly with lower thresholds, although, as mentioned above, there was no correlation between the number of whisks emitted in a trial and acuity.

We also compared the power spectra of correct and incorrect trials for all (except NAIVE and C2) task conditions. The average PSDs for correct and incorrect trials were slightly but significantly different in parts of the whisking frequency range (Fig. $12 E)$. Although there was no difference at frequencies below $5 \mathrm{~Hz}$ and above $14 \mathrm{~Hz}$, the spectral power was significantly lower ( $p=0.002-0.04$, Wilcoxon's test) between 5-8 and 11-14 Hz in incorrect compared with correct trials. This finding is consistent with the observation that performance levels improved with an increase in whisking spectral power.

\section{Discussion}

We characterized the acuity and behavior of rats during a horizontal object localization task. Whereas rats with all, a single row, or a single arc of whiskers intact learned to localize, rats with only a single whisker intact on each cheek from training onset did not learn to localize objects reliably. However, rats trained to localize with multiple whiskers could later localize with a single whisker intact. Typically, the performance of rats initially trained with multiple whiskers improved after trimming all but one whisker on each cheek. During localization, rats actively whisked. Although we found no relationship between whisking frequency and performance, there was a strong correlation between performance and the total whisking spectral power in the range of 5-25 Hz: as spectral power increased, thresholds were lowered.
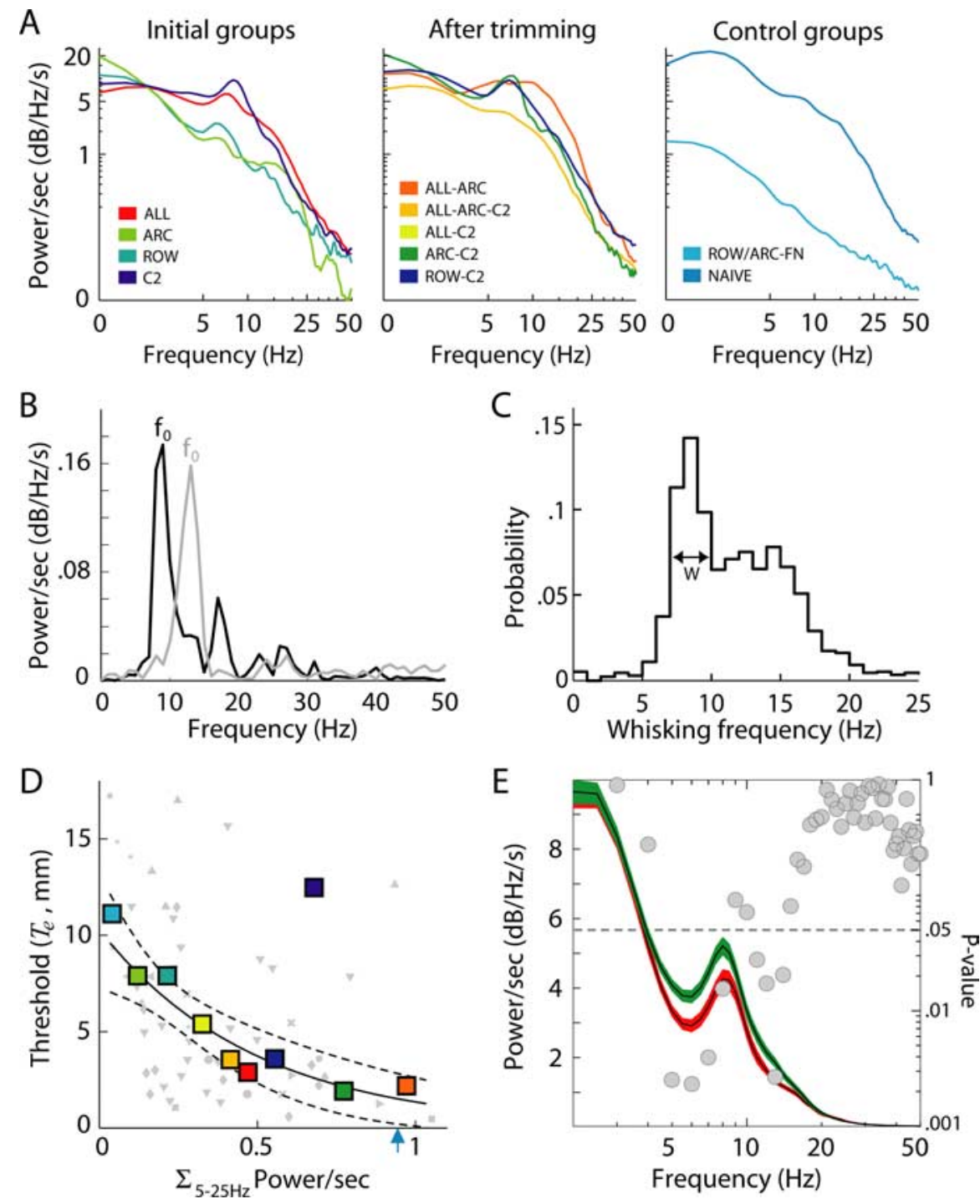

Figure 12. Whisking and performance. A, PSDs of whisker angle during task performance. PSDs (power per second) were computed for individual trials, normalized with respect to time, and averaged. Trials (44-783) of two, three, or four rats were tracked for each condition (total of $62 \mathrm{~min}$ ). B, PSDs of angular velocities of the whisker trajectories depicted in Figure 8, C (black) and $D$ (gray). The whisking frequency is defined as the peak of the PSD $\left(f_{0}\right)$. C, Probability distribution of whisking frequencies of rats localizing objects (data from all whisker configurations; $n=1945$ ). Trials were excluded if the maximal value of the PSD was less than its mean +2 SDs. D, Acuity and net power (power per second) in the range of 5-25 Hz. Colors indicate whisker configuration (same as in $\boldsymbol{A}$ ). Small symbols show the average power computed across trials of an individual testing session and its threshold. Large symbols show the average power and threshold computed across all trials and sessions of a configuration. The solid line shows the best fit of the exponential function $\alpha \times e^{-\beta P}$ to the data points of individual sessions, and dashed lines show its $95 \%$ confidence interval. The C 2 configuration, based on its position after averaging across sessions, was considered an outlier and not included in the fit. The blue arrow indicates the average whisking power of naive rats. $E$, PSDs of whisker angles of all trials averaged across whisker configurations, except C2, according to trial outcome. Incorrect trials are shown in red, and correct trials are in green. The two PSDs were compared at $1 \mathrm{~Hz}$ bins (Wilcoxon's test); gray circles indicate the $p$ values of the differences. The dotted line indicates the 0.05 significance level.

\section{Learning}

Although rats failed to acquire the localization task if tested with a single whisker intact from the onset of training, they continued to make fine horizontal discriminations if they had initially been trained with all whiskers and then were tested with a single whisker. This paradoxical result could be explained by stress, sensory confusion, or behavioral effects related to whisker removal. Sensory deprivation associated with trimming all but a pair of whiskers may cause physiological stress that can negatively impact task acquisition more than task performance. However, we found no difference in the time it took rats with a single or multiple whiskers to acquire the procedural aspects of the task. Thus, the learning impairment and the possible influence of physiological stress were specific to localization only. The degree of sensory deprivation was, however, related to performance levels during initial training. Rats with all whiskers intact (ALL) reached lower thresholds than rats with a single row (ROW) or arc (ARC), ROW and ARC rats performed similarly, and ROW and ARC rats performed better than the single whisker (C2) group. Still, the majority of rats improved after the initial whisker removal, such as in the groups ALL-C2, ALL- 
ARC, ALL-ARC-C2, ROW-C2, and ARC-C2. Thus, sensory deprivation appears to have been detrimental only to task learning but not to performance potential.

The relationship between a reduction in number of whiskers and learning could be related to how reliably the behavior of the rats encoded critical sensory parameters. For example, as the number of intact whiskers is reduced, spatial cues, such as the identities of contacting whiskers, are diminished. The correlation between performance and the number of intact whiskers in the initial training conditions (ALL, ARC, ROW, and C2) might reflect a dependency on spatial cues for efficient learning of the task. Interestingly, although spatial information may be necessary for learning, it is clearly not required to perform the task, because rats with a single pair of intact whiskers could perform the task.

\section{Limits of horizontal object localization by vibrissal touch}

Rats trained to discriminate radial apertures reach thresholds of $\sim 3 \mathrm{~mm}$ (Krupa et al., 2001). We found that rats discriminating relative horizontal offsets can reach thresholds as low as $0.24 \mathrm{~mm}$. This offset corresponds to $\sim 1^{\circ}$ difference between the left and right $\mathrm{C} 2$ whiskers and is in the range of the minimal whisker displacement that rats can detect (Hutson and Masterton, 1986). If horizontal location was encoded by the identities of contacting and noncontacting whiskers along a row, then localization acuity should be limited by the inter-vibrissal separation. However, horizontal localization thresholds were as low as $\sim 1 / 10$ of the whisker spacing. Such spatial sensitivity is not unique to the vibrissal system. Human subjects can assign the relative location of a visual stimulus with a precision that is finer than the spacing of photoreceptors in the fovea, an ability known as hyperacuity (Westheimer, 1981). Hence, we propose the term vibrissal hyperacuity to describe the ability of rats to discriminate positional offsets smaller then the inter-vibrissal distance. Despite considerable intersubject variability in humans, visual hyperacuity is typically attained in every testing session once practiced with a particular stimulus configuration (Fahle and Edelman, 1993). Here, not all rats achieved vibrissal hyperacuity, and those that did, did not reach hyperacuity levels in all testing sessions (Table 2). We speculate that this difference between human visual hyperacuity and rat vibrissal hyperacuity could be attributed to larger behavioral variability (motor and sensory), confusion about the task, or changes in motivation and attention.

Vibrissal hyperacuity cannot be achieved by a whisker-identity code alone, because hyperacuity also requires a spatial representation of the region between neighboring whiskers. A moving whisker maps the inter-vibrissal region directly, and afferents may encode horizontal location by signaling the elapsed interval between whisking onset and contact (Szwed et al., 2003). Thus, temporal encoding could facilitate vibrissal hyperacuity. Temporal encoding also does not require multiple whiskers, suggesting that it may be used by single-whisker rats. The inter-vibrissal region could also be specified by an intensity code derived from the forces acting on the follicle as a result of bending of the whisker as it contacts an object: the more posterior an object, the more the whisker will bend (assuming the attempted whisking amplitude remains the same). Whatever singlewhisker cues were used by our rats, our results indicate that they enable hyperacuity, as shown by the ALL-ARC-C2 group, and can be pooled along an arc to additionally increase acuity, as shown by our ALL-ARC rats.

\section{Relationship between whisking and acuity}

During exploration, rats actively move their whiskers back and forth in a stereotypic manner. The exact behavioral relevance of this behavior for sensory encoding is unclear. The mystacial pad, like the retina or the finger pad, is an example of a spatially distributed detector array. Whiskers are embedded in highly innervated folliclesinus complexes (FSCs) (Ebara et al., 2002), and the mechanoreceptors that detect vibrissal touch are located longitudinal to the FSC. Thus, the spatial resolution of a static whisker array is limited by the inter-vibrissal spacing. To differentiate small spatial offsets, the region between the whiskers must be scanned by moving the whiskers through it. The specialized whisking behavior of rats suggests that whisking is part of a specific encoding strategy. Szwed et al. (2003) recently showed that trigeminal (TG) neurons can encode variations in horizontal location in the range of hyperacuity during active whisking in anesthetized rats, suggesting a role of whisker movements in resolving small positional offsets. Here, we found that whisking behavior (net power in the whisking range) indeed correlated with performance levels. We also found that rats with lesioned facial motor nerves could not correctly localize objects along the horizontal axis. Thus, whisker movements are necessary and beneficiary in a graded manner for horizontal localization.

Both behavioral and neuronal observations suggest that different encoding schemes exist for radial and horizontal object location. Although horizontal acuity can be improved by moving the whiskers, this advantage does not apply for the radial dimension (along the length of the whiskers). In fact, rats performing a radial discrimination task appear to suppress whisking (Krupa et al., 2001). Also, single-whisker rats, even those pretrained with multiple whiskers, cannot make correct radial discriminations (Krupa et al., 2001), although, as we show here, they can make accurate horizontal discriminations. These behavioral differences of object localization are consistent with how the horizontal and radial dimensions are encoded by TG neurons: whereas the radial coordinate of an object is encoded by the spiking rate of TG neurons, the horizontal location is best encoded by TG spike timing (Szwed et al., 2003, 2006).

\section{Implications of whisker trimming for sensory processing}

We found that sensory deprivation, through whisker trimming, could lead to an improvement in horizontal localization acuity. Whisker trimming is known to exert physiological changes throughout the trigeminal pathway (Diamond et al., 1993; Kelly et al., 1999; Polley et al., 1999; Harwell et al., 2005; Zuo et al., 2005), and behavioral changes (such as localization acuity) could result from these changes. Alternatively, behavioral changes could result from the constraints imposed by the new configuration of the whisker array. For instance, removing all but one whisker may lead to an increase in whisking intensity, because a single whisker now has to cover a region of space previously covered by many whiskers. This constraint on whisking behavior may lead to different, possibly more optimal, motor-sensory strategies being used during localization. In a previous study on tactile discrimination of textured surfaces, we found that human observers improved their performance if instructed to emphasize, or unknowingly were restricted to, temporal cues (Gamzu and Ahissar, 2001). Whereas multiple whiskers provide an animal with both spatial and temporal information, a single whisker cannot provide spatial information. This, combined with the observation that rats actively move their whiskers during horizontal localization, suggests that rats, as humans, may emphasize temporal strategies when spatial information is lacking. We proposed previously how active movements and temporal encoding could 
facilitate hyperacuity performance (Ahissar and Arieli, 2001). Here, we show that rats reach vibrissal hyperacuity and that such performance is associated with active whisker movements in the absence of multiwhisker (spatial) cues. Although several sensory cues reliably encode horizontal offsets, it is uncertain at present which components of the tactile input guide horizontal object localization during learning or performance.

\section{References}

Ahissar E, Arieli A (2001) Figuring space by time. Neuron 32:185-201.

Berg RW, Kleinfeld D (2003a) Rhythmic whisking by rat: retraction as well as protraction of the vibrissae is under active muscular control. J Neurophysiol 89:104-117.

Berg RW, Kleinfeld D (2003b) Vibrissa movement elicited by rhythmic electrical microstimulation to motor cortex in the aroused rat mimics exploratory whisking. J Neurophysiol 90:50-63.

Bermejo R, Houben D, Zeigler HP (1998) Optoelectronic monitoring of individual whisker movements in rats. J Neurosci Methods 83:89-96.

Brecht M, Preilowski B, Merzenich MM (1997) Functional architecture of the mystacial vibrissae. Behav Brain Res 84:81-97.

Carvell GE, Simons DJ (1990) Biometric analyses of vibrissal tactile discrimination in the rat. J Neurosci 10:2638-2648.

Carvell GE, Simons DJ (1995) Task- and subject-related differences in sensorimotor behavior during active touch. Somatosens Mot Res 12:1-9.

Carvell GE, Simons DJ, Lichtenstein SH, Bryant P (1991) Electromyographic activity of mystacial pad musculature during whisking behavior in the rat. Somatosens Mot Res 8:159-164.

Carvell GE, Miller SA, Simons DJ (1996) The relationship of vibrissal motor cortex unit activity to whisking in the awake rat. Somatosens Mot Res 13:115-127

Diamond ME, Armstrong-James M, Ebner FF (1993) Experiencedependent plasticity in adult rat barrel cortex. Proc Natl Acad Sci USA 90:2082-2086.

Dorfl J (1982) The musculature of the mystacial vibrissae of the white mouse. J Anat 135:147-154.

Ebara S, Kumamoto K, Matsuura T, Mazurkiewicz JE, Rice FL (2002) Similarities and differences in the innervation of mystacial vibrissal folliclesinus complexes in the rat and cat: a confocal microscopic study. J Comp Neurol 449:103-119.

Fahle M, Edelman S (1993) Long-term learning in vernier acuity: effects of stimulus orientation, range and of feedback. Vision Res 33:397-412.

Fee MS, Mitra PP, Kleinfeld D (1997) Central versus peripheral determinants of patterned spike activity in rat vibrissa cortex during whisking. J Neurophysiol 78:1144-1149.

Gamzu E, Ahissar E (2001) Importance of temporal cues for tactile spatialfrequency discrimination. J Neurosci 21:7416-7427.

Gao P, Bermejo R, Zeigler HP (2001) Whisker deafferentation and rodent whisking patterns: behavioral evidence for a central pattern generator. J Neurosci 21:5374-5380.

Green DM, Swets JA (1966) Signal detection theory and psychophysics. New York: Wiley.

Guic-Robles E, Valdivieso C, Guajardo G (1989) Rats can learn a roughness discrimination using only their vibrissal system. Behav Brain Res 31:285-289.

Hamada Y, Miyashita E, Tanaka H (1999) Gamma-band oscillations in the "barrel cortex" precede rat's exploratory whisking. Neuroscience 88:667-671.

Harvey MA, Bermejo R, Zeigler HP (2001) Discriminative whisking in the head-fixed rat: optoelectronic monitoring during tactile detection and discrimination tasks. Somatosens Mot Res 18:211-222.

Harwell C, Burbach B, Svoboda K, Nedivi E (2005) Regulation of cpg15 expression during single whisker experience in the barrel cortex of adult mice. J Neurobiol 65:85-96.

Hutson KA, Masterton RB (1986) The sensory contribution of a single vibrissa's cortical barrel. J Neurophysiol 56:1196-1223.

Jenkinson EW, Glickstein M (2000) Whiskers, barrels, and cortical efferent pathways in gap crossing by rats. J Neurophysiol 84:1781-1789.
Kelly DH (1979) Motion and vision. I. Stabilized images of stationary gratings. J Opt Soc Am 69:1266-1274.

Kelly MK, Carvell GE, Kodger JM, Simons DJ (1999) Sensory loss by selected whisker removal produces immediate disinhibition in the somatosensory cortex of behaving rats. J Neurosci 19:9117-9125.

Knutsen PM, Derdikman D, Ahissar E (2005) Tracking whisker and head movements in unrestrained behaving rodents. J Neurophysiol 93:2294-2301.

Krupa DJ, Matell MS, Brisben AJ, Oliveira LM, Nicolelis MA (2001) Behavioral properties of the trigeminal somatosensory system in rats performing whisker-dependent tactile discriminations. J Neurosci 21:5752-5763.

Lamb GD (1983) Tactile discrimination of textured surfaces: psychophysical performance measurements in humans. J Physiol (Lond) 338:551-565.

Nicolelis MA, Baccala LA, Lin RC, Chapin JK (1995) Sensorimotor encoding by synchronous neural ensemble activity at multiple levels of the somatosensory system. Science 268:1353-1358.

O'Connor SM, Berg RW, Kleinfeld D (2002) Coherent electrical activity between vibrissa sensory areas of cerebellum and neocortex is enhanced during free whisking. J Neurophysiol 87:2137-2148.

Perret S, Noble W (1997) The contribution of head motion cues to localization of low-pass noise. Percept Psychophys 59:1018-1026.

Polley DB, Chen-Bee CH, Frostig RD (1999) Two directions of plasticity in the sensory-deprived adult cortex. Neuron 24:623-637.

Polley DB, Rickert JL, Frostig RD (2005) Whisker-based discrimination of object orientation determined with a rapid training paradigm. Neurobiol Learn Mem 83:134-142.

Rice FL, Munger BL (1986) A comparative light microscopic analysis of the sensory innervation of the mystacial pad. II. The common fur between the vibrissae. J Comp Neurol 252:186-205.

Rice JA (1995) Mathematical statistics and data analysis, Ed 2. Belmont, CA: Duxbury.

Rucci M, Desbordes G (2003) Contributions of fixational eye movements to the discrimination of briefly presented stimuli. J Vis 3:852-864.

Sachdev RN, Sato T, Ebner FF (2002) Divergent movement of adjacent whiskers. J Neurophysiol 87:1440-1448.

Sachdev RN, Berg RW, Champney G, Kleinfeld D, Ebner FF (2003) Unilateral vibrissa contact: changes in amplitude but not timing of rhythmic whisking. Somatosens Mot Res 20:163-169.

Semba K, Komisaruk BR (1984) Neural substrates of two different rhythmical vibrissal movements in the rat. Neuroscience 12:761-774.

Shuler MG, Krupa DJ, Nicolelis MA (2002) Integration of bilateral whisker stimuli in rats: role of the whisker barrel cortices. Cereb Cortex 12:86-97.

Szwed M, Bagdasarian K, Ahissar E (2003) Encoding of vibrissal active touch. Neuron 40:621-630.

Szwed M, Bagdasarian K, Blumenfeld B, Barak O, Derdikman D, Ahissar E (2006) Responses of trigeminal ganglion neurons to the radial distance of contact during active vibrissal touch. J Neurophysiol 95:791-802.

Tulunay-Keesey U, VerHoeve JN (1987) The role of eye movements in motion detection. Vision Res 27:747-754.

Vincent S (1912) The function of the vibrissae in the behavior of the white rat. Behav Monograph 1:7-81.

Wallach H (1940) The role of head movements and vestibular and visual cues in sound localization. J Exp Psychol 27:339-368.

Welker WI (1964) Analysis of sniffing of the albino rat. Behavior 22:223-244.

Westheimer G (1981) Visual hyperacuity. Berlin: Springer.

Wightman FL, Kistler DJ (1999) Resolution of front-back ambiguity in spatial hearing by listener and source movement. J Acoust Soc Am 105:2841-2853

Wineski LE (1983) Movements of the cranial vibrissae in the golden hamster. J Zool 200:261-280.

Wineski LE (1985) Facial morphology and vibrissal movement in the golden hamster. J Morphol 183:199-217.

Zuo Y, Yang G, Kwon E, Gan WB (2005) Long-term sensory deprivation prevents dendritic spine loss in primary somatosensory cortex. Nature 436:261-265. 